\title{
DISCREPANCY OF MINIMAL RIESZ ENERGY POINTS
}

\author{
JORDI MARZO AND ALBERT MAS
}

\begin{abstract}
We find upper bounds for the spherical cap discrepancy of the set of minimizers of the Riesz $s$-energy on the sphere $\mathbb{S}^{d}$. Our results are based in bounds for a Sobolev discrepancy introduced by Thomas Wolff in an unpublished manuscript where estimates for the spherical cap discrepancy of the logarithmic energy minimizers in $\mathbb{S}^{2}$ were obtained. Our result improves previously known bounds for $0 \leq s<2$ and $s \neq 1$ in $\mathbb{S}^{2}$, where $s=0$ is Wolff's result, and for $d-t_{0}<s<d$ with $t_{0} \approx 2.5$ when $d \geq 3$ and $s \neq d-1$.
\end{abstract}

\section{INTRODUCTION AND MAIN RESULTS}

For an $N$ point set $X_{N}=\left\{x_{1}, \ldots, x_{N}\right\}$ in the unit sphere $\mathbb{S}^{d}=\left\{x \in \mathbb{R}^{d+1}:|x|=1\right\}$ and $0 \leq s<d$ the Riesz $s$-energy of $X_{N}$ is given by

$$
E_{s}\left(X_{N}\right)=\sum_{i \neq j} \frac{1}{\left|x_{i}-x_{j}\right|^{s}}, \quad \text { if } 0<s<d,
$$

and

$$
E_{0}\left(X_{N}\right)=\sum_{i \neq j} \log \frac{1}{\left|x_{i}-x_{j}\right|}, \quad \text { if } s=0 .
$$

We denote the minimal Riesz $s$-energy, for $0 \leq s<d$, achived by an $N$ point set by

$$
\mathcal{E}_{s}(N)=\inf _{X_{N}} E_{s}\left(X_{N}\right),
$$

where $X_{N}$ runs through the $N$ point sets $X_{N} \subset \mathbb{S}^{d}$.

Problems related to these minimal energies or with the minimizers, in the spherical and in other settings, have been extensively studied. It is well known that the continuous Riesz $s$-energy of the surface measure on the sphere gives the leading term of the asymptotic expansion of the normalized discrete energy. Moreover, due to the work of different authors, see [7, 8] and references therein, it is known that for $d \geq 2$ and $0<s<d$ there exist constants $C, c>0$ such that

$$
-c N^{1+s / d} \leq \mathcal{E}_{s}(N)-E_{s}(\widetilde{\sigma}) N^{2} \leq-C N^{1+s / d}
$$

Key words and phrases. Minimal energy points, Spherical cap discrepancy, Sobolev discrepancy.

The authors are grateful to Joaquim Ortega-Cerdà and Carlos Beltrán for enlightening discussions on the subject matter of this paper, and to Alexandre Eremenko for information about Wolff's manuscript.

The first author has been supported by grant MTM2017-83499-P by the Ministerio de Economía y Competitividad, Gobierno de España and by the Generalitat de Catalunya (project 2017 SGR 358). The second author was partially supported by MTM2017-84214 and MTM2017-83499 projects of the MCINN (Spain), 2017-SGR-358 project of the AGAUR (Catalunya), and ERC-2014-ADG project HADE Id. 669689 (European Research Council). 
for $N \geq 2$, where

$$
E_{s}(\widetilde{\sigma})=\int_{\mathbb{S}^{d}} \int_{\mathbb{S}^{d}} \frac{1}{|x-y|^{s}} d \widetilde{\sigma}(x) d \widetilde{\sigma}(y)=2^{d-s-1} \frac{\Gamma\left(\frac{d+1}{2}\right) \Gamma\left(\frac{d-s}{2}\right)}{\sqrt{\pi} \Gamma\left(d-\frac{s}{2}\right)}
$$

and $\widetilde{\sigma}$ is the normalized surface measure in $\mathbb{S}^{d}$ given by the relation $\sigma=\omega_{d} \widetilde{\sigma}$, being $\sigma$ the surface measure in the sphere and $\omega_{d}=\sigma\left(\mathbb{S}^{d}\right)=2 \pi^{\frac{d+1}{2}} / \Gamma\left(\frac{d+1}{2}\right)$. For the logarithmic case $s=0$ it is known that

$$
\mathcal{E}_{0}(N)=E_{0}(\widetilde{\sigma}) N^{2}-\frac{1}{d} N \log N+O(N),
$$

where

$$
E_{0}(\widetilde{\sigma})=\int_{\mathbb{S}^{d}} \int_{\mathbb{S}^{d}} \log \frac{1}{|x-y|} d \widetilde{\sigma}(x) d \widetilde{\sigma}(y)=\frac{\psi_{0}(d)-\psi_{0}(d / 2)}{2}-\log 2
$$

and $\psi_{0}$ denotes the digamma function.

Several conjectures about the lower order terms on these asymptotic expansions and, in some particular dimensions, about the value of the constants appearing in the asymptotic expansion can be found in the literature, see [2, 4, 7, 8, 24].

There are still many basic open questions about the distribution of the minimizers. Recall that $X_{N}=\left\{x_{1}, \ldots, x_{N}\right\} \subset \mathbb{S}^{d}$ is separated if

$$
\min _{i \neq j}\left|x_{i}-x_{j}\right| \geq C N^{-\frac{1}{d}}
$$

for some constant $C>0,[8$. The minimizers are known to be separated if $0 \leq s<2$ for $d=2$ and $d-2 \leq s<d$ for $d \geq 3$, but in $0 \leq s<d-2$ and $d \geq 3$, the best bound is $O\left(N^{-\frac{1}{s+2}}\right),[10,12,13,[19$.

It is classical that, for any $0 \leq s<d$, minimizers of the Riesz $s$-energy in $\mathbb{S}^{d}$ are asymptotically uniformly distributed, meaning that

$$
\lim _{N \rightarrow+\infty} \frac{1}{N} \sum_{j=1}^{N} f\left(x_{j}\right)=\int_{\mathbb{S}^{d}} f(x) d \widetilde{\sigma}(x), \quad \text { for all } f \in \mathcal{C}\left(\mathbb{S}^{d}\right),
$$

or, equivalently, that the sum of delta measures $\mu_{N}=\frac{1}{N} \sum_{j=1}^{N} \delta_{x_{j}}$ converges in the weak-* topology to the normalized surface measure $\widetilde{\sigma}$. It is also a well known fact that the $N$ point sets $\left\{X_{N}\right\}_{N}$ are asymptotically uniformly distributed if and only if the spherical cap discrepancy converges to zero

$$
\lim _{N \rightarrow+\infty} \sup _{x \in \mathbb{S}^{d}, r>0}\left|\frac{\#\left(X_{N} \cap D_{r}(x)\right)}{N}-\widetilde{\sigma}\left(D_{r}(x)\right)\right|=0,
$$

where $D_{r}(x)=\left\{y \in \mathbb{S}^{d}:|x-y|<r\right\}$ is a spherical cap of center $x \in \mathbb{S}^{d}$ and (euclidean) distance $r>0$. Loosely speaking, the speed of this convergence is a measure of how well distributed are the points in $\left\{X_{N}\right\}_{N}$.

Our objective is to provide upper bounds for the spherical cap discrepancy of $N$ point sets of minimizers of the Riesz s-energy. Previous work around this problem focused basically in the Coulomb potential case $s=d-1$. This case is somehow 
simpler because, for $d \geq 2,|x|^{1-d}$ is (modulo a constant) the fundamental solution for the Laplacian in $\mathbb{R}^{d+1}$

$$
-\Delta\left(|x|^{1-d}\right)=(d-1) \omega_{d} \delta_{0}
$$

where $\delta_{0}$ is a Dirac delta at the origin. Points which minimize the $(d-1)$-energy are called Fekete points and, in this setting, the results are tipically valid for regular enough $d$-dimensional surfaces in $\mathbb{R}^{d+1}$, not only for $\mathbb{S}^{d}$. The first result is due to Kleiner [16] and yields that the spherical cap discrepancy of a set of Fekete points on the sphere is $O\left(N^{-\frac{1}{3 d}}\right)$. Sjögren [26] improved this result to $O\left(N^{-\frac{1}{2 d}}\right)$. In 1996 Korevaar [17] conjectured that the right bound was $O\left(N^{-\frac{1}{d}}\right)$. Finally, Götz [15] proved Korevaar's conjecture, up to a logarithmic factor, giving the best known result $O\left(N^{-\frac{1}{d}} \log N\right)$.

For all other cases, $s \neq d-1$, the only known results are due to Brauchart, who established the bound

$$
O\left(N^{-\frac{d-s}{d(d-s+2)}}\right) \text {, if } 0 \leq s<d,
$$

see [5] for $0<s<d$ and [6] for the logarithmic case. Observe that, in the harmonic case $s=d-1$, Brauchart's result gives a bound of the same order as Kleiner's.

However, for the logarithmic case in $\mathbb{S}^{2}$, Wolff proved in an unpublished manuscript [29] the bound $O\left(N^{-1 / 3}\right)$, which is better than the $O\left(N^{-1 / 4}\right)$ following from Brauchart (1.4). For more information about Wolff's manuscript see Remark 4.3. Our objective in this work is to generalize Wolff's approach to other $s$-energies in $\mathbb{S}^{d}$. In this regard, our main result is an upper bound for the spherical cap discrepancy of the energy minimizers that improves Brauchart's result in the range $0 \leq s<2$ for $d=2$ (where $s=0$ is Wolff's result) and in the range $d-t_{0}<s<d$, for $d \geq 3$, where $t_{0}=\frac{1+\sqrt{17}}{2}>2$. In all these cases, when $s=d-1$ Götz's mentioned result is still the best one.

Theorem 1.1. Let $0 \leq s<d$ and $X_{N}=\left\{x_{1}, \ldots, x_{N}\right\}$ be an $N$-point set of minimizers of the Riesz s-energy in $\mathbb{S}^{d}$. Then

$$
\sup _{D}\left|\frac{\#\left(X_{N} \cap D\right)}{N}-\widetilde{\sigma}(D)\right| \lesssim \chi_{[0, d-2]}(s) N^{-\frac{2}{d(d-s+1)}}+\chi_{(d-2, d)}(s) N^{-\frac{2(d-s)}{d(d-s+4)}}
$$

with constants depending only on $d$ and $s$, where the supremum on the left hand side runs over all spherical caps $D \subset \mathbb{S}^{d}$.

Remark 1.2. The same bound above holds when the discrepancy is defined in terms of the so called $K$-regular sets instead of the spherical caps, [26].

Observe that, in the harmonic case $s=d-1$, our result gives a bound between Kleiner's and Sjögren's results. Note also that all these results, ours and Götz's, are far from the optimal spherical cap discrepancy established by Beck for $N$ point sets in $\mathbb{S}^{d}$, which is of order $N^{-\frac{d+1}{2 d}}$, up to a logarithmic term, [1].

Theorem 1.1 gives a quantitative proof of the asymptotic equidistribution of the energy minimizers. It would be interesting to extend our approach to Green energies on manifods like the ones studied in [3].

Following Wolff's approach, Theorem 1.1 on the spherical cap discrepancy will follow from a sharp estimate of a discrepancy defined in terms of Sobolev norms. We will introduce now the needed concepts. 
1.1. Spherical harmonics and Sobolev discrepancy. Given an integer $\ell \geq 0$, let $\mathcal{H}_{\ell}$ be the vector space of the spherical harmonics of degree $\ell$, i.e. the space of eigenfunctions of the the Laplace-Beltrami operator $\Delta$ on $\mathbb{S}^{d}$,

$$
-\Delta Y=\ell(\ell+d-1) Y, \quad Y \in \mathcal{H}_{\ell},
$$

of eigenvalue $\ell(\ell+d-1)$. The value $h_{\ell}=\operatorname{dim} \mathcal{H}_{\ell}$, is the multiplicity of the eigenvalue $\ell(\ell+d-1)$ and it is easily seen to be $h_{\ell} \approx \ell^{d-1}$.

For the Hilbert space $L^{2}\left(\mathbb{S}^{d}\right)$ of square integrable functions in $\mathbb{S}^{d}$ with the inner product

$$
\langle f, g\rangle=\int_{\mathbb{S}^{d}} f(x) g(x) d \sigma(x), \quad f, g \in L^{2}\left(\mathbb{S}^{d}\right),
$$

one has the decomposition $L^{2}\left(\mathbb{S}^{d}\right)=\bigoplus_{\ell \geq 0} \mathcal{H}_{\ell}$. Therefore, for $f \in L^{2}\left(\mathbb{S}^{d}\right)$, one has the Fourier representation

$$
f=\sum_{\ell, k} f_{\ell, k} Y_{\ell, k}, \quad f_{\ell, k}=\left\langle f, Y_{\ell, k}\right\rangle=\int_{\mathbb{S}^{d}} f Y_{\ell, k} d \sigma
$$

where $\left\{Y_{\ell, k}\right\}_{k=1}^{h_{\ell}}$ is an orthonormal basis of $\mathcal{H}_{\ell}$.

Given $r \geq 0$ we consider the standard $L^{2}\left(\mathbb{S}^{d}\right)$-based Sobolev spaces of order $r$ defined in terms of their representation on the Fourier side, namely,

$$
\mathbb{H}^{r}\left(\mathbb{S}^{d}\right)=\left\{f \in L^{2}\left(\mathbb{S}^{d}\right): \sum_{\ell=0}^{+\infty} \sum_{k=1}^{h_{\ell}}\left(1+\ell^{2}\right)^{r}\left|f_{\ell, k}\right|^{2}<+\infty\right\},
$$

with the norm

$$
\|f\|_{\mathbb{H}^{r}\left(\mathbb{S}^{d}\right)}=\left(\sum_{\ell=0}^{+\infty} \sum_{k=1}^{h_{\ell}}\left(1+\ell^{2}\right)^{r}\left|f_{\ell, k}\right|^{2}\right)^{1 / 2}
$$

Since $\left(1+\ell^{2}\right)^{r},\left(1+\ell^{r}\right)^{2}$, and $1+\ell^{2 r}$ are comparable for all $\ell \geq 0$ with constants only depending on $r$, in the sequel we may use at our convenience any of these expressions to estimate the norm $\|\cdot\|_{\mathbb{H}^{r}\left(\mathbb{S}^{d}\right)}$. Recall that $\mathbb{H}^{r}\left(\mathbb{S}^{d}\right)$ is continuously embedded in $\mathcal{C}^{k}\left(\mathbb{S}^{d}\right)$ if $r-k>d / 2$.

For any Borel measure $\mu$ in $\mathbb{S}^{d}$, we consider a "dual" Sobolev norm of $\mu$ defined by

$$
\|\mu\|_{\mathbb{H}^{-r}\left(\mathbb{S}^{d}\right)}=\sup \left\{\int_{\mathbb{S}^{d}} \psi d \mu: \psi \in \mathcal{C}^{\infty}\left(\mathbb{S}^{d}\right),\|\psi\|_{\mathbb{H}^{r}\left(\mathbb{S}^{d}\right)}=1\right\} .
$$

When the measure is of the form $\mu=h \sigma$ for some $h \in L^{2}\left(\mathbb{S}^{d}\right)$, by an abuse of notation we will simply write $\|h\|_{\mathbb{H}-r(\mathbb{S} d)}$.

Following [21, 29], we define the following discrepancy with respect to functions in the Sobolev space.

Definition 1.3. Let $X_{N}=\left\{x_{1}, \ldots, x_{N}\right\}$ be a set of $N$ points on the sphere $\mathbb{S}^{d}$. Given $\epsilon>0$ and $0 \leq s<d$, the Sobolev discrepancy of $X_{N}$ is

$$
D_{s, d}^{\epsilon}\left(X_{N}\right)=\left\|\mu_{X_{N}, \epsilon}\right\|_{\mathbb{H}^{\frac{s-d}{2}}\left(\mathbb{S}^{d}\right)},
$$


where

$$
\mu_{X_{N}, \epsilon}=\left(\frac{1}{N} \sum_{j=1}^{N} \frac{\chi_{D_{j}}}{\sigma\left(D_{j}\right)}-\frac{1}{\omega_{d}}\right) \sigma,
$$

and $D_{j}=D_{\epsilon N^{-1 / d}}\left(x_{j}\right)$.

Remark 1.4. In [29] Wolff considered a homogenous Sobolev norm instead. But both in the original work of Wolff and in the present article, these norms are used to pass from the spherical cap discrepancy (an $L^{\infty}$ estimate) to the Sobolev discrepancy (a "dual" Sobolev estimate), and then to the asymptotics of the energy. One can check in the proof of Theorem 1.1 below that the zero order term, say $\|f\|_{L^{2}\left(\mathbb{S}^{d}\right)}$, can be absorbed by the dominant term and our final conclusion completely agrees with the one of Wolff.

Our following result is an estimate of the Sobolev discrepancy of minimizers that is sharp in the range $d-2 \leq s<d$.

Theorem 1.5. Let $0 \leq s<d$ and $X_{N}=\left\{x_{1}, \ldots, x_{N}\right\}$ be an $N$ point set of minimizers of the Riesz s-energy in $\mathbb{S}^{d}$. Then, for every $\epsilon>0$ small enough depending only on $d$ and $s$,

$$
N^{-\frac{1}{2}+\frac{s}{2 d}} \lesssim D_{s, d}^{\epsilon}\left(X_{N}\right) \lesssim N^{-\frac{1}{d}}+N^{-\frac{1}{2}+\frac{s}{2 d}}
$$

with constants depending only $d, s$, and $\epsilon$.

Remark 1.6. It is well known that the linearization of the quadratic Wasserstein distance is precisely the homogenous Sobolev norm considered by Wolff (see Remark 1.4) [27, 7.6]. Moreover, Peyre has recently shown that the quadratic Wasserstein distance is bounded above by the the homogenous Sobolev norm and therefore Wolff's result can be read in terms of a quadratic Wasserstein discrepancy, [22].

1.2. Notation. Given $d \geq 2$ integer, we denote by $\Delta$ and $\nabla$ the spherical Laplacian and spherical gradient on $\mathbb{S}^{d} \subset \mathbb{R}^{d+1}$, respectively. Given $s \geq 0$ and two different points $x, y \in \mathbb{R}^{d+1}$, let the Riesz kernel of order $s$ acting on $(x, y)$ be defined by

$$
R_{s}(x, y):= \begin{cases}|x-y|^{-s}, & \text { for } s>0 \\ -\log |x-y|, & \text { for } s=0 .\end{cases}
$$

For $0 \leq s<d$ and $f \in L^{2}\left(\mathbb{S}^{d}\right)$, we define the spherical Riesz transform of $f$ by

$$
R_{s} f(x):=\int_{\mathbb{S}^{d}} R_{s}(x, y) f(y) d \sigma(y), \quad \text { for } x \in \mathbb{S}^{d} .
$$

We denote the Riesz s-energy of a Borel measure $\mu$ in $\mathbb{S}^{d}$ by

$$
E_{s}(\mu):=\int_{\mathbb{S}^{d}} \int_{\mathbb{S}^{d}} R_{s}(x, y) d \mu(y) d \mu(x) .
$$

When $\mu=f \sigma$ with $f \in L^{2}\left(\mathbb{S}^{d}\right)$, we write $E_{s}(f)$ instead of $E_{s}(f \sigma)$, hence

$$
E_{s}(f)=\int_{\mathbb{S}^{d}} f(x) R_{s} f(x) d \sigma(x)=\int_{\mathbb{S}^{d}} \int_{\mathbb{S}^{d}} R_{s}(x, y) f(x) f(y) d \sigma(y) d \sigma(x) .
$$


1.3. Structure of the article. Section 2 contains the preliminaries. In there, we set the basic properties of the Riesz kernels and the spherical Riesz transform, namely, we show that the operator $R_{s}$ diagonalizes in the standard basis of spherical harmonics, we find its eigenvalues in a closed form and their asymptotic behavior, and we study the relation between the Riesz kernels and the Laplace-Beltrami operator on the sphere, giving some heuristics.

Section 3 focuses on asymptotic estimates of Riesz energies on the sphere. The main result is an estimate of the continuous Riesz energy of small discs centered at the discrete minimizers in terms of the minimal energy $\mathcal{E}_{s}(N)$. This, together with the asymptotic expansion of the minimal energy, is a key tool to derive the estimates of the Sobolev discrepancy given in Theorem 1.5, which is proven in Section 4.

Finally, in Section [5 we give the proof of Theorem [1.1, which is a straightforward application of Theorem 1.5 and Proposition 5.2.

\section{Spectral analysis of the Spherical Riesz transform}

In this section we set basic properties of the Riesz kernels and the spherical Riesz transform. On one hand, we show that the operator $R_{s}$ diagonalizes in the standard basis of spherical harmonics. In addition, we find its eigenvalues in a closed form using hypergeometric functions, and we analyze their asymptotic behavior. This is the purpose of Proposition 2.2, a key result that will be systematically used in the sequel. In particular, it allows us to relate in Lemma 2.4 below the Riesz energies $E_{s}$ to the dual Sobolev norms $\|\cdot\|_{\mathbb{H}^{(s-d) / 2}\left(\mathbb{S}^{d}\right)}$ for $0 \leq s<d$.

On the other hand, we explore the relation between the Riesz kernels and the Laplace-Beltrami operator on the sphere. Essentially, we show that the kernel $R_{s+2}$ can be obtained by applying the Helmoltz differential operator $-\Delta+C_{d, s}$ to the kernel $R_{s}$, where $C_{d, s}$ is a suitable constant depending on $d$ and $s$; see Lemma 2.5 for the precise statement. In the particular case of $s=d-2>0$, we get that $R_{d-2}$ is a multiple of a fundamental solution of $-\Delta+(d-2) d / 4$. These identities are the departing point in Section 3 to get the asymptotic estimates for Riesz energies.

Even though the asymptotic behavior of the eigenvalues for the spherical Riesz transform is obtained in Proposition 2.2 for the whole range $0 \leq s<d$ by a direct argument, it is of interest to see how one can get it in the subcritical regime $(0<s<$ $d-2)$ from its knowledge in the critical $(s=d-2)$ and supercritical $(d-2<s<$ d) regimes by an iteration argument based on the connection between $R_{s}$ and $R_{s+2}$ mentioned before. We develop this argument at the end of this section. This served us to see how, for every positive integer $m$, the Sobolev norms $\|\cdot\|_{\mathbb{H}^{m}\left(\mathbb{S}^{d}\right)}$ defined in terms of the spherical harmonics decomposition correspond to the standard Sobolev norms given by pure derivatives, and gave us an intuition for extending Wolff's arguments for $(s, d)=(0,2)$ to the whole range $0 \leq s<d$. These last considerations are treated in Lemma 2.7.

2.1. Fourier multipliers. This part is devoted to show that the spherical harmonics diagonalize the spherical Riesz transform, and to find the asymptotic behavior of the eigenvalues. With this at hand, we find a simple expression in terms of the Fourier coefficients which serves to connect the Riesz energy to a dual Sobolev norm. 
For the expression of the Riesz potential and the Riesz energy of a function $f \in$ $L^{2}\left(\mathbb{S}^{d}\right)$ written in terms of spherical harmonics, we recall the following definition of a generalized hypergeometric function.

Definition 2.1. For integers $p, q \geq 0$ and complex values $a_{i}, b_{j}$, the generalized hypergeometric function is defined by the power series

$$
{ }_{p} F_{q}\left(a_{1}, \ldots, a_{p} ; b_{1}, \ldots, b_{q} ; z\right)=\sum_{n=0}^{\infty} \frac{\left(a_{1}\right)_{n} \ldots\left(a_{p}\right)_{n}}{\left(b_{1}\right)_{n} \ldots\left(b_{q}\right)_{n}} \frac{z^{n}}{n !},
$$

where $(\cdot)_{n}$ is the rising factorial or Pochhammer symbol given by $(x)_{0}=1$ and

$$
(x)_{n}=x(x+1) \cdots(x+n-2)(x+n-1)=\frac{\Gamma(x+n)}{\Gamma(x)}, \quad n \geq 1,
$$

for $x \in \mathbb{C}$.

Proposition 2.2. Let $0 \leq s<d$ and let $\left\{Y_{\ell, k}\right\}_{\ell, k}$ for $\ell=0,1, \ldots$ and $k=1, \ldots h_{\ell}$ be an orthonormal basis of spherical harmonics in $L^{2}\left(\mathbb{S}^{d}\right)$. Given $f \in L^{2}\left(\mathbb{S}^{d}\right)$, we have

$$
R_{s} f(x)=\int_{\mathbb{S}^{d}} R_{s}(x, y) f(y) d \sigma(y)=\sum_{\ell, k} A_{\ell, s} f_{\ell, k} Y_{\ell, k}(x)
$$

for almost all $x \in \mathbb{S}^{d}$, and

$$
E_{s}(f)=\int_{\mathbb{S}^{d}} \int_{\mathbb{S}^{d}} R_{s}(x, y) f(x) f(y) d \sigma(y) d \sigma(x)=\sum_{\ell, k} A_{\ell, s}\left|f_{\ell, k}\right|^{2},
$$

where $f_{\ell, k}=\int_{\mathbb{S}^{d}} f Y_{\ell, k} d \sigma$ and

$$
\begin{aligned}
A_{\ell, s} & =\frac{2^{d-s} \Gamma\left(\frac{d-s}{2}\right)}{\Gamma\left(d-\frac{s}{2}\right)}{ }_{3} F_{2}\left(-\ell, \ell+d-1, \frac{d-s}{2} ; \frac{d}{2}, d-\frac{s}{2} ; 1\right) \\
& =\frac{2^{d-s} \Gamma\left(\frac{d-s}{2}\right) \Gamma\left(\frac{s}{2}+\ell\right)}{\Gamma\left(\frac{s}{2}\right) \Gamma\left(d-\frac{s}{2}+\ell\right)} .
\end{aligned}
$$

Additionally, there exists $C>0$ only depending on $s$ and $d$ such that

$$
\frac{C^{-1}}{1+\ell^{d-s}} \leq A_{\ell, s} \leq \frac{C}{1+\ell^{d-s}} \quad \text { for all } \ell \geq 0
$$

In particular,

$$
E_{s}(f) \approx \sum_{\ell, k} \frac{1}{1+\ell^{d-s}}\left|f_{\ell, k}\right|^{2}
$$

Proof. We first consider the case $s>0$. If we set $F_{s}(t)=(2-2 t)^{-s / 2}$, then $R_{s}(x, y)=$ $F_{s}(\langle x, y\rangle)$. By Funk-Hecke formula, see [11, page 11],

$$
\int_{\mathbb{S}^{d}} F_{s}(\langle x, y\rangle) Y_{\ell, k}(x) d \sigma(x)=\frac{\omega_{d-1}}{C_{\ell}^{\frac{d-1}{2}}(1)}\left(\int_{-1}^{1} F_{s}(t) C_{\ell}^{\frac{d-1}{2}}(t)\left(1-t^{2}\right)^{\frac{d-2}{2}} d t\right) Y_{\ell, k}(y),
$$


and the expression for $R_{s} f(x)$ follows if we set

$$
A_{\ell, s}=\frac{\omega_{d-1}}{2^{s / 2} C_{\ell}^{\frac{d-1}{2}}(1)} \int_{-1}^{1} C_{\ell}^{\frac{d-1}{2}}(t)(1-t)^{\frac{d-s}{2}-1}(1+t)^{\frac{d-2}{2}} d t .
$$

The expression for $E_{s}(f)$ follows then by orthogonality, that is,

$$
\int_{\mathbb{S}^{d}} \int_{\mathbb{S}^{d}} R_{s}(x, y) Y_{\ell, k}(x) Y_{\ell^{\prime}, k^{\prime}}(y) d \sigma(x) d \sigma(y)=A_{\ell, s} \delta_{(\ell, k),\left(\ell^{\prime}, k^{\prime}\right)} .
$$

From [14, page 281] one can get a closed expression in terms of an hypergeometric function

$$
\begin{aligned}
& \int_{-1}^{1} C_{\ell}^{\frac{d-1}{2}}(t)(1-t)^{\frac{d-s}{2}-1}(1+t)^{\frac{d-2}{2}} d t \\
& \quad=2^{d-\frac{s}{2}-1} \frac{\Gamma\left(\frac{d-s}{2}\right) \Gamma\left(\frac{d}{2}\right) \Gamma(\ell+d-1)}{\Gamma(\ell+1) \Gamma(d-1) \Gamma\left(d-\frac{s}{2}\right)}{ }_{3} F_{2}\left(-\ell, \ell+d-1, \frac{d-s}{2} ; \frac{d}{2}, d-\frac{s}{2} ; 1\right) .
\end{aligned}
$$

From Saalshütz's theorem we get

$$
{ }_{3} F_{2}\left(-\ell, \ell+d-1, \frac{d-s}{2} ; \frac{d}{2}, d-\frac{s}{2} ; 1\right)=\frac{\Gamma\left(\frac{s}{2}+\ell\right) \Gamma\left(d-\frac{s}{2}\right)}{\Gamma\left(\frac{s}{2}\right) \Gamma\left(d-\frac{s}{2}+\ell\right)},
$$

which yields (2.2).

Finally, the asymptotic expression for the quotient of gamma functions

$$
\lim _{n \rightarrow+\infty} \frac{\Gamma(n+\alpha)}{\Gamma(n) n^{\alpha}}=1, \quad \alpha \in \mathbb{C},
$$

proves (2.3) because, clearly, $A_{\ell, s} \neq 0$.

The endpoint case $s=0$ is obtained using $F_{0}(t)=-\frac{1}{2} \log (2-2 t)$ and taking the derivative with respect to $s$ and evaluating at $s=0$ the expression (2.5).

Remark 2.3. From Proposition 2.2 it follows that, at a formal level,

$$
\begin{aligned}
\frac{1}{|x-y|^{s}} & =\sum_{\ell, k} A_{\ell, s} Y_{\ell, k}(x) Y_{\ell, k}(y)=\sum_{\ell} A_{\ell, s} \sum_{k} Y_{\ell, k}(x) Y_{\ell, k}(y) \\
& =\sum_{\ell} \frac{A_{\ell, s}}{\omega_{d}} \frac{2 \ell+d-1}{d-1} C_{\ell}^{\frac{d-1}{2}}(\langle x, y\rangle),
\end{aligned}
$$

where $\langle x, y\rangle$ is the cosine of the angle between $x$ and $y$, and $C_{\ell}^{\alpha}(t)$ is the Gegenbauer polynomial orthogonal in $[-1,1]$ with respect to $\left(1-t^{2}\right)^{\alpha-\frac{1}{2}}$ with the normalization $C_{\ell}^{\alpha}(1)=\left(\begin{array}{c}2 \alpha+k-1 \\ k\end{array}\right)$. But as

$$
\frac{A_{\ell, s}}{\omega_{d}}=\frac{2^{d-s-1} \Gamma\left(\frac{d+1}{2}\right) \Gamma\left(\frac{d-s}{2}\right) \Gamma\left(\frac{s}{2}+\ell\right)}{\sqrt{\pi} \Gamma\left(\frac{s}{2}\right) \Gamma\left(d-\frac{s}{2}+\ell\right)},
$$

we get that $A_{\ell, d-1}=\omega_{d}(d-1) /(2 \ell+d-1)$ and, thus,

$$
\frac{1}{|x-y|^{d-1}}=\sum_{\ell} C_{\ell}^{\frac{d-1}{2}}(\langle x, y\rangle) \text {. }
$$


In particular, it is well known that for the Newtonian potential and $x, y \in \mathbb{S}^{2}$ one has

$$
\frac{1}{|x-y|}=\sum_{\ell} P_{\ell}(\langle x, y\rangle),
$$

where $P_{\ell}(t)$ is the Legendre polynomial of degree $\ell$ normalized as $P_{\ell}(1)=1$.

Using Proposition 2.2, in the following result we highlight the important connection between $\|\cdot\|_{\mathbb{H}^{(s-d) / 2}\left(\mathbb{S}^{d}\right)}$ and the Riesz $s$-energy $E_{s}$ introduced in (1.7).

Lemma 2.4. Given $0 \leq s<d$, there exists a constant $C>0$ only depending on $s$ and $d$ such that, for every $h \in L^{2}\left(\mathbb{S}^{d}\right)$,

$$
C^{-1}\|h\|_{\mathbb{H}^{(s-d) / 2}\left(\mathbb{S}^{d}\right)}^{2} \leq E_{s}(h) \leq C\|h\|_{\mathbb{H}^{(s-d) / 2}\left(\mathbb{S}^{d}\right)}^{2} .
$$

Proof. By a limiting argument, it suffices to prove the lemma when $h$ is smooth. On one hand, in (2.4) we showed that if $h=\sum_{\ell, k} h_{\ell, k} Y_{\ell, k}$ then

$$
E_{s}(h) \approx \sum_{\ell, k} \frac{1}{1+\ell^{d-s}}\left|h_{\ell, k}\right|^{2} .
$$

Writing every $\psi \in \mathcal{C}^{\infty}\left(\mathbb{S}^{d}\right)$ as $\psi=\sum_{\ell, k} \psi_{\ell, k} Y_{\ell, k}$, by Cauchy-Schwarz inequality we have

$$
\begin{aligned}
\left|\int_{\mathbb{S}^{d}} \psi h d \sigma\right|^{2} & =\left|\sum_{\ell, k} h_{\ell, k} \psi_{\ell, k}\right|^{2} \leq\left(\sum_{\ell, k} \frac{\left|h_{\ell, k}\right|^{2}}{1+\ell^{d-s}}\right)\left(\sum_{\ell, k}\left(1+\ell^{d-s}\right)\left|\psi_{\ell, k}\right|^{2}\right) \\
& \approx E_{s}(h)\|\psi\|_{\mathbb{H}^{(d-s) / 2}\left(\mathbb{S}^{d}\right)}^{2},
\end{aligned}
$$

which yields the first inequality in (2.6) by taking the supremum on $\psi \in \mathcal{C}^{\infty}\left(\mathbb{S}^{d}\right)$.

Let us now prove the second inequality. Since $h$ is smooth by assumption, it is not hard to see that indeed $R_{s} h$ too. Thanks to (2.1) and (2.3) we have that $R_{s} h=$ $\sum_{\ell, k} A_{\ell, s} h_{\ell, k} Y_{\ell, k}$ with $A_{\ell, s} \approx\left(1+\ell^{d-s}\right)^{-1}$. Hence, we can estimate

$$
\begin{aligned}
E_{s}(h) & =\int_{\mathbb{S}^{d}} h R_{s} h d \sigma \leq\|h\|_{\mathbb{H}^{(s-d) / 2}\left(\mathbb{S}^{d}\right)}\left\|R_{s} h\right\|_{\mathbb{H}^{(d-s) / 2}\left(\mathbb{S}^{d}\right)} \\
& \approx\|h\|_{\mathbb{H}^{(s-d) / 2}\left(\mathbb{S}^{d}\right)}\left(\sum_{\ell, k}\left(1+\ell^{d-s}\right)\left|A_{\ell, s} h_{\ell, k}\right|^{2}\right)^{1 / 2} \approx\|h\|_{\mathbb{H}^{(s-d) / 2}\left(\mathbb{S}^{d}\right)} E_{s}(h)^{1 / 2},
\end{aligned}
$$

and the second inequality in (2.6) follows.

2.2. The Laplace-Beltrami operator on the Riesz kernel. In this section we give useful identities which connect the Riesz kernels of different indexes through the Laplace-Beltrami operator. All of them are collected in the following lemma, which will be systematically used in Section 3 to get the asymptotic estimates for Riesz energies.

Lemma 2.5. Let $x_{0} \in \mathbb{S}^{d}$. Then for $d \geq 2$ and $s>0$, as a function of $x \in \mathbb{S}^{d}$,

$$
\left(-\Delta+\frac{1}{4} s(2 d-2-s)\right) R_{s}\left(x, x_{0}\right)=s(d-2-s) R_{s+2}\left(x, x_{0}\right), \quad \text { for all } x \neq x_{0} .
$$

Furthermore, in the special case of $d>2$ and $s=d-2$ we have

$$
\left(-\Delta+\frac{1}{4}(d-2) d\right) R_{d-2}\left(\cdot, x_{0}\right)=C_{d}(d-2) \delta_{x_{0}}
$$


in the sense of distributions, where $\delta_{x_{0}}$ denotes the Dirac measure at $x_{0}$ and $C_{d}=$ $2 \pi^{d / 2} / \Gamma(d / 2)$. Indeed, for every open set $\Omega \subset \mathbb{S}^{d}$ with smooth boundary and such that $x_{0} \in \Omega$, and every $f \in \mathcal{C}^{2}(\Omega) \cap \mathcal{C}^{1}(\bar{\Omega})$, we have

$$
\begin{aligned}
C_{d}(d-2) f\left(x_{0}\right)= & \int_{\Omega} R_{d-2}\left(x, x_{0}\right)\left(-\Delta+\frac{1}{4}(d-2) d\right) f(x) d \sigma(x) \\
& +\int_{\partial \Omega}\left(R_{d-2}\left(x, x_{0}\right) \nabla f(x)-f(x) \nabla R_{d-2}\left(x, x_{0}\right)\right) \cdot \nu(x) d \sigma^{\prime}(x),
\end{aligned}
$$

where $\sigma^{\prime}$ denotes the $(d-1)$-dimensional Hausdorff measure.

In the logarithmic case $s=0$ and $d>2$, we have

$$
-\Delta R_{0}\left(x, x_{0}\right)=(d-2) R_{2}\left(x, x_{0}\right)-\frac{d-1}{2}, \quad \text { for all } x \neq x_{0},
$$

and, when $s=0$ and $d=2$,

$$
-\Delta R_{0}\left(\cdot, x_{0}\right)=2 \pi \delta_{x_{0}}-\frac{1}{2}
$$

in the sense of distributions. That is, for every open set $\Omega \subset \mathbb{S}^{2}$ with smooth boundary and such that $x_{0} \in \Omega$, and every $f \in \mathcal{C}^{2}(\Omega) \cap \mathcal{C}^{1}(\bar{\Omega})$, we have

$$
\begin{aligned}
2 \pi f\left(x_{0}\right)= & -\int_{\Omega} R_{0}\left(x, x_{0}\right) \Delta f(x) d \sigma(x)+\frac{1}{2} \int_{\Omega} f(x) d \sigma(x) \\
& +\int_{\partial \Omega}\left(R_{0}\left(x, x_{0}\right) \nabla f(x)-f(x) \nabla R_{0}\left(x, x_{0}\right)\right) \cdot \nu(x) d \sigma^{\prime}(x) .
\end{aligned}
$$

Proof. Let $\Delta_{\mathbb{R}^{d+1}}$ denote the standard Laplacian in $\mathbb{R}^{d+1}$ and $\pi: \mathbb{R}^{d+1} \backslash\{0\} \rightarrow \mathbb{S}^{d}$ be the spherical projection given by $\pi(y)=y /|y|$ for $y \in \mathbb{R}^{d+1} \backslash\{0\}$. It is well known that if $f: \mathbb{S}^{d} \rightarrow \mathbb{R}$ then the spherical Laplacian of $f$ can be computed through $\Delta_{\mathbb{R}^{d+1}}$ by the formula

$$
\Delta f(x)=\left(\Delta_{\mathbb{R}^{d+1}}(f \circ \pi)\right)(\pi(y))
$$

at the points $x=\pi(y)$ where $f \circ \pi$ is twice differentiable.

We will consider the case $s>0$, the logarithmic case $s=0$ follows easily along the same lines. Take $x_{0} \in \mathbb{S}^{d}$. A computation shows that, as a function of $y \in \mathbb{R}^{d+1} \backslash\{0\}$,

$$
\begin{aligned}
& \Delta_{\mathbb{R}^{d+1}}\left(R_{s}\left(\pi(\cdot), x_{0}\right)\right)(y) \\
& \quad=s\left\{(s+2) R_{s+4}\left(\pi(y), x_{0}\right) f\left(\frac{\left|x_{0}\right|^{2}}{|y|^{2}}-\frac{\left(y \cdot x_{0}\right)^{2}}{|y|^{4}}\right)-d R_{s+2}\left(\pi(y), x_{0}\right) \frac{\left(y \cdot x_{0}\right)}{|y|^{3}}\right\} .
\end{aligned}
$$

Using the definition of $R_{s}$, that $x=\pi(y)$ and that $\left|x_{0}\right|=|x|=1$, we then get

$$
\begin{aligned}
& \Delta_{\mathbb{R}^{d+1}}\left(R_{s}(\right.\left.\left.\pi(\cdot), x_{0}\right)\right)(\pi(y)) \\
& \quad=s\left\{(s+2) R_{s+4}\left(x, x_{0}\right)\left(1-\left(x \cdot x_{0}\right)^{2}\right)-d R_{s+2}\left(x, x_{0}\right)\left(x \cdot x_{0}\right)\right\} \\
& \quad=s\left|x-x_{0}\right|^{-s-2}\left\{(s+2) \frac{1-\left(x \cdot x_{0}\right)^{2}}{\left|x-x_{0}\right|^{2}}-d\left(x \cdot x_{0}\right)\right\} .
\end{aligned}
$$


Observe that

$$
1-\left(x \cdot x_{0}\right)^{2}=\left(1+\left(x \cdot x_{0}\right)\right)\left(1-\left(x \cdot x_{0}\right)\right)=\left(1+\left(x \cdot x_{0}\right)\right) \frac{\left|x-x_{0}\right|^{2}}{2} .
$$

Therefore,

$$
\begin{aligned}
\Delta_{\mathbb{R}^{d+1}}\left(R_{s}\left(\pi(\cdot), x_{0}\right)\right) & (\pi(y))=s\left|x-x_{0}\right|^{-s-2}\left\{\frac{s+2}{2}\left(1+\left(x \cdot x_{0}\right)\right)-d\left(x \cdot x_{0}\right)\right\} \\
& =\frac{s}{2}\left|x-x_{0}\right|^{-s-2}\left\{(2 d-2-s)\left(1-\left(x \cdot x_{0}\right)\right)+2(s+2-d)\right\} \\
& =\frac{s}{2}\left|x-x_{0}\right|^{-s-2}\left\{(2 d-2-s) \frac{\left|x-x_{0}\right|^{2}}{2}+2(s+2-d)\right\} \\
& =\frac{s}{4}(2 d-2-s) R_{s}\left(x, x_{0}\right)+s(s+2-d) R_{s+2}\left(x, x_{0}\right)
\end{aligned}
$$

which, together with (2.12), yields (2.7). For the logarithmic case $s=0$, the previous computations lead to

$$
\begin{aligned}
\Delta_{\mathbb{R}^{d+1}}\left(R_{0}\left(\pi(\cdot), x_{0}\right)\right)(\pi(y)) & =\frac{1}{2}\left|x-x_{0}\right|^{-2}\left\{(2 d-2) \frac{\left|x-x_{0}\right|^{2}}{2}+2(2-d)\right\} \\
& =\frac{d-1}{2}+(2-d) R_{2}\left(x, x_{0}\right),
\end{aligned}
$$

which proves (2.10).

We now address (2.8). Given $x_{0} \in \Omega \subset \mathbb{S}^{d}$ and $\epsilon>0$ set $\Omega_{\epsilon}:=\Omega \backslash D_{\epsilon}\left(x_{0}\right)$, whose boundary is the disjoint union of $\partial \Omega$ and $\partial D_{\epsilon}\left(x_{0}\right)$ if $\epsilon$ is small enough. We denote by $\nu$ the outward unit normal vector (tangent to $\mathbb{S}^{d}$ ) on $\partial \Omega_{\epsilon}$. An integration by parts gives

$$
\begin{aligned}
\int_{\Omega_{\epsilon}} R_{d-2}\left(x, x_{0}\right)\left(-\Delta+\frac{1}{4}(d-2) d\right) f(x) d \sigma(x) \\
=\int_{\Omega_{\epsilon}} \nabla R_{d-2}\left(x, x_{0}\right) \cdot \nabla f(x) d \sigma(x)-\int_{\partial \Omega_{\epsilon}} R_{d-2}\left(x, x_{0}\right) \nabla f(x) \cdot \nu(x) d \sigma^{\prime}(x) \\
\quad+\frac{1}{4}(d-2) d \int_{\Omega_{\epsilon}} R_{d-2}\left(x, x_{0}\right) f(x) d \sigma(x) \\
=\int_{\partial \Omega_{\epsilon}} \nabla R_{d-2}\left(x, x_{0}\right) \cdot \nu(x) f(x) d \sigma^{\prime}(x)-\int_{\partial \Omega_{\epsilon}} R_{d-2}\left(x, x_{0}\right) \nabla f(x) \cdot \nu(x) d \sigma^{\prime}(x) \\
\quad+\int_{\Omega_{\epsilon}}\left(-\Delta+\frac{1}{4}(d-2) d\right) R_{d-2}\left(x, x_{0}\right) f(x) d \sigma(x) .
\end{aligned}
$$

Since dist $\left(x_{0}, \Omega_{\epsilon}\right)>0$, the last term on the right hand side of (2.14) vanishes by (2.7). Note also that

$$
\begin{aligned}
& \mid \int_{\partial D_{\epsilon}\left(x_{0}\right)} R_{d-2}\left(x, x_{0}\right) \nabla f(x) \cdot \nu(x) d \sigma^{\prime}(x) \mid \\
& \leq\|\nabla f\|_{L^{\infty}(\Omega)} \epsilon^{-d+2} \sigma\left(\partial D_{\epsilon}\left(x_{0}\right)\right)=O(\epsilon) .
\end{aligned}
$$


Arguing as in (2.12), we have that $\nabla R_{d-2}\left(x, x_{0}\right)=-(d-2) R_{d}\left(x, x_{0}\right)\left(\left(x \cdot x_{0}\right) x-x_{0}\right)$. Moreover, for $x \in \partial D_{\epsilon}\left(x_{0}\right)$ the outward unit normal vector with respect to $\Omega_{\epsilon} \subset \mathbb{S}^{d}$ is

$$
\nu(x)=\frac{x_{0}-x-\left(\left(x_{0}-x\right) \cdot x\right) x}{\left|x_{0}-x \cdot x-\left(\left(x_{0}-x\right) \cdot x\right) x\right|}=\frac{x_{0}-\left(x_{0} \cdot x\right) x}{\left|x_{0}-\left(x_{0} \cdot x\right) x\right|},
$$

which leads to $\nabla R_{d-2}\left(x, x_{0}\right) \cdot \nu(x)=(d-2) R_{d}\left(x, x_{0}\right)\left|x_{0}-\left(x_{0} \cdot x\right) x\right|$. Observe also that, from (2.13),$\left|x_{0}-\left(x_{0} \cdot x\right) x\right|^{2}=1-\left(x_{0} \cdot x\right)^{2}=\frac{1}{2}\left(1+\left(x_{0} \cdot x\right)\right)\left|x-x_{0}\right|^{2}$. Therefore,

$$
\begin{aligned}
\int_{\partial D_{\epsilon}\left(x_{0}\right)} \nabla R_{d-2}\left(x, x_{0}\right) \cdot \nu(x) & f(x) d \sigma^{\prime}(x) \\
= & \frac{d-2}{\epsilon^{d-1}} \int_{\partial D_{\epsilon}\left(x_{0}\right)}\left(\frac{1+\left(x_{0} \cdot x\right)}{2}\right)^{1 / 2} f(x) d \sigma^{\prime}(x) .
\end{aligned}
$$

Since the integrand is continuous near $x_{0}$, we deduce that

$$
\lim _{\epsilon \rightarrow 0} \int_{\partial D_{\epsilon}\left(x_{0}\right)} \nabla R_{d-2}\left(x, x_{0}\right) \cdot \nu(x) f(x) d \sigma^{\prime}(x)=C_{d}(d-2) f\left(x_{0}\right),
$$

where

$$
C_{d}:=\lim _{\epsilon \rightarrow 0} \epsilon^{1-d} \sigma^{\prime}\left(\partial D_{\epsilon}\left(x_{0}\right)\right)=\frac{2 \pi^{d / 2}}{\Gamma\left(\frac{d}{2}\right)} .
$$

Finally, taking the limit $\epsilon \rightarrow 0$ in (2.14) and using (2.15) and (2.17) we get (2.9). The statement in (2.8) is a consequence of (2.9) taking $\Omega=\mathbb{S}^{d}$, thus $\partial \Omega=\emptyset$.

Observe that in the logarithmic case $\nabla R_{0}\left(x, x_{0}\right)=-R_{2}\left(x, x_{0}\right)\left(\left(x \cdot x_{0}\right) x-x_{0}\right)$, and then formula (2.16) becomes

$$
\int_{\partial D_{\epsilon}\left(x_{0}\right)} \nabla R_{0}\left(x, x_{0}\right) \cdot \nu(x) f(x) d \sigma^{\prime}(x)=\frac{1}{\epsilon} \int_{\partial D_{\epsilon}\left(x_{0}\right)}\left(\frac{1+\left(x_{0} \cdot x\right)}{2}\right)^{1 / 2} f(x) d \sigma^{\prime}(x) .
$$

Hence, the same argument yields the case $d=2$.

2.3. From the supercritical to the subcritical regime through iteration. By a direct argument, in Proposition 2.2 we found the asymptotic behavior of the eigenvalues for the Spherical Riesz transform for the whole range $0 \leq s<d$, see (2.3). However, it is of interest to see how one can get it in the subcritical regime from its knowledge in the critical and supercritical regimes by an iteration argument based on (2.7). This is the purpose of this section.

We begin by showing, directly from (2.8), the asymptotics (2.3) in the critical regime $0<s=d-2$. Given $f \in L^{2}\left(\mathbb{S}^{d}\right)$ set $f_{\ell, k}=\int f Y_{\ell, k} d \sigma$, hence (2.1) gives $R_{s} f=$ $\sum_{\ell, k} A_{\ell, s} f_{\ell, k} Y_{\ell, k}$. Thanks to (2.8) and (1.5) we get

$$
\begin{aligned}
\sum_{\ell, k} f_{\ell, k} Y_{\ell, k} & =f=\frac{1}{C_{d}(d-2)}\left(-\Delta+\frac{1}{4}(d-2) d\right) R_{d-2} f \\
& =\frac{1}{C_{d}(d-2)}\left(-\Delta+\frac{1}{4}(d-2) d\right) \sum_{\ell, k} A_{\ell, d-2} f_{\ell, k} Y_{\ell, k} \\
& =\sum_{\ell, k} f_{\ell, k} \frac{A_{\ell, d-2}}{C_{d}}\left(\frac{\ell(\ell+d-1)}{d-2}+\frac{d}{4}\right) Y_{\ell, k} .
\end{aligned}
$$


Since this holds for all $f \in L^{2}\left(\mathbb{S}^{d}\right)$ we deduce that

$$
A_{\ell, d-2}=C_{d}\left(\frac{\ell(\ell+d-1)}{d-2}+\frac{d}{4}\right)^{-1}
$$

and (2.3) follows in this case.

Assuming now that (2.3) holds in the (super)critical regime $0<d-2 \leq s<d$, let us deal with the case $s \in(0, d-2)$. Let $m \in \mathbb{N}$ be such that $s \in[d-2(m+1), d-2 m)$, thus indeed $m$ is the unique integer such that $(d-s) / 2-1 \leq m<(d-s) / 2$. Then $s+2 m \in[d-2, d)$ and, by assumption,

$$
A_{\ell, s+2 m} \approx \frac{1}{1+\ell^{d-s-2 m}} \quad \text { for all } \ell \geq 0 .
$$

Furthermore, if we set $s_{j}=s+2 j$ for $j=0,1,2, \ldots, m$, iterating (2.7) we deduce that

$$
\begin{aligned}
R_{s_{m}}(\cdot, x) & =\frac{-\Delta+s_{m-1}\left(\frac{d-1}{2}-\frac{s_{m-1}}{4}\right)}{s_{m-1}\left(d-2-s_{m-1}\right)} R_{s_{m-1}}(\cdot, x) \\
& =\frac{-\Delta+s_{m-1}\left(\frac{d-1}{2}-\frac{s_{m-1}}{4}\right)}{s_{m-1}\left(d-2-s_{m-1}\right)} \cdots \frac{-\Delta+s_{0}\left(\frac{d-1}{2}-\frac{s_{0}}{4}\right)}{s_{0}\left(d-2-s_{0}\right)} R_{s_{0}}(\cdot, x)
\end{aligned}
$$

Then, similarly to what we did in (2.18), from (2.1), (2.20), and (1.5) we have

$$
\begin{aligned}
& \sum_{\ell, k} A_{\ell, s+2 m} f_{\ell, k} Y_{\ell, k}=R_{s_{m}} f \\
&= \frac{-\Delta+s_{m-1}\left(\frac{d-1}{2}-\frac{s_{m-1}}{4}\right)}{s_{m-1}\left(d-2-s_{m-1}\right)} \cdots \frac{-\Delta+s_{0}\left(\frac{d-1}{2}-\frac{s_{0}}{4}\right)}{s_{0}\left(d-2-s_{0}\right)} R_{s_{0}} f \\
&= \frac{-\Delta+s_{m-1}\left(\frac{d-1}{2}-\frac{s_{m-1}}{4}\right)}{s_{m-1}\left(d-2-s_{m-1}\right)} \cdots \frac{-\Delta+s_{0}\left(\frac{d-1}{2}-\frac{s_{0}}{4}\right)}{s_{0}\left(d-2-s_{0}\right)} \sum_{\ell, k} A_{\ell, s} f_{\ell, k} Y_{\ell, k} \\
&= \sum_{\ell, k} A_{\ell, s} \frac{\ell(\ell+d-1)+s_{m-1}\left(\frac{d-1}{2}-\frac{s_{m-1}}{4}\right)}{s_{m-1}\left(d-2-s_{m-1}\right)} \\
& \cdots \frac{\ell(\ell+d-1)+s_{0}\left(\frac{d-1}{2}-\frac{s_{0}}{4}\right)}{s_{0}\left(d-2-s_{0}\right)} f_{\ell, k} Y_{\ell, k} .
\end{aligned}
$$

This combined to (2.19) leads to

$$
\begin{aligned}
A_{\ell, s} & =A_{\ell, s+2 m} \frac{s_{m-1}\left(d-2-s_{m-1}\right)}{\ell(\ell+d-1)+s_{m-1}\left(\frac{d-1}{2}-\frac{s_{m-1}}{4}\right)} \cdots \frac{s_{0}\left(d-2-s_{0}\right)}{\ell(\ell+d-1)+s_{0}\left(\frac{d-1}{2}-\frac{s_{0}}{4}\right)} \\
& \approx \frac{1}{\left(1+\ell^{d-s-2 m}\right)\left(1+\ell^{2 m}\right)} \approx \frac{1}{1+\ell^{d-s}}
\end{aligned}
$$

for all $\ell \geq 0$, and (2.3) follows in the subcritical regime.

Remark 2.6. From the previous computations, if $d>2$ we can get the explicit expressions

$$
A_{\ell, d-2}=\frac{2 \pi^{d / 2}}{\Gamma(d / 2)} \cdot \frac{1}{\frac{\ell(\ell+d-1)}{d-2}+\frac{d}{4}}
$$


and

$$
A_{\ell, d-2 k}=A_{\ell, d-2} \frac{2}{\frac{\ell(\ell+d-1)}{d-4}+\frac{d+2}{4}} \cdot \frac{4}{\frac{\ell(\ell+d-1)}{d-6}+\frac{d+4}{4}} \cdots \frac{2(k-1)}{\frac{\ell(\ell+d-1)}{d-2 k}+\frac{d+2(k-1)}{4}}
$$

for all $k \geq 2$ integer such that $d-2 k>0$. A similar formula can be shown for $A_{\ell, 0}$ if $d=4,6,8, \ldots$ by taking into account (2.10) to pass from $A_{\ell, 0}$ to $A_{\ell, 2}$. We omit the details.

2.4. The connection to Sobolev spaces on the sphere. The computations in Section 2.3 served us to see how, for every positive integer $m$, the Sobolev norms $\|\cdot\|_{\mathbb{H}^{m}\left(\mathbb{S}^{d}\right)}$ defined in terms of the spherical harmonics decomposition correspond to the standard Sobolev norms given by pure derivatives, giving us an intuition for extending Wolff's arguments for the case $(s, d)=(0,2)$ to the whole range $0 \leq s<d$. In order to clarify this, let us first make some considerations on the Sobolev spaces $\mathbb{H}^{m}\left(\mathbb{S}^{d}\right)$. Of course, $\mathbb{H}^{0}\left(\mathbb{S}^{d}\right)=L^{2}\left(\mathbb{S}^{d}\right)$. Looking at the spherical harmonics, for every given $j \in \mathbb{N}$ an integration by parts and (1.5) show that

$$
\int_{\mathbb{S}^{d}}\left|\Delta^{j} Y_{\ell, k}\right|^{2} d \sigma=\int_{\mathbb{S}^{d}} Y_{\ell, k} \Delta^{2 j} Y_{\ell, k} d \sigma=\ell^{2 j}(\ell+d-1)^{2 j} \approx \ell^{4 j}
$$

and

$$
\int_{\mathbb{S}^{d}}\left|\nabla \Delta^{j} Y_{\ell, k}\right|^{2} d \sigma=-\int_{\mathbb{S}^{d}} Y_{\ell, k} \Delta^{2 j+1} Y_{\ell, k} d \sigma=\ell^{2 j+1}(\ell+d-1)^{2 j+1} \approx \ell^{4 j+2}
$$

for all $\ell \geq 0$, with constants only depending on $d$ and $j$. Moreover, by the orthogonality of the basis $\left\{Y_{\ell, k}\right\}_{k=1, \ell \geq 0}^{h_{\ell}}$, we also get

$$
\int_{\mathbb{S}^{d}} Y_{\ell, k} \Delta^{j} Y_{\ell^{\prime}, k^{\prime}} d \sigma=0
$$

for all $j \in \mathbb{N} \cup\{0\}$ whenever $(\ell, k) \neq\left(\ell^{\prime}, k^{\prime}\right)$.

Given an odd number $m \in \mathbb{N}$ set $i=(m-1) / 2$. Then, using (1.6), (2.21), (2.22), and (2.23), for every $f \in L^{2}\left(\mathbb{S}^{d}\right)$ we see that

$$
\begin{aligned}
\|f\|_{\mathbb{H}^{m}\left(\mathbb{S}^{d}\right)}^{2} & \approx \sum_{\ell=0}^{+\infty} \sum_{k=1}^{h_{\ell}}\left(1+\ell^{2 m}\right)\left|f_{\ell, k}\right|^{2} \approx \sum_{\ell=0}^{+\infty} \sum_{k=1}^{h_{\ell}} \sum_{j=0}^{i}\left(\ell^{4 j}+\ell^{4 j+2}\right)\left|f_{\ell, k}\right|^{2} \\
& \approx \sum_{j=0}^{i} \sum_{\ell=0}^{+\infty} \sum_{k=1}^{h_{\ell}}\left(\int_{\mathbb{S}^{d}} Y_{\ell, k} \Delta^{2 j} Y_{\ell, k} d \sigma-\int_{\mathbb{S}^{d}} Y_{\ell, k} \Delta^{2 j+1} Y_{\ell, k} d \sigma\right)\left|f_{\ell, k}\right|^{2} \\
& =\sum_{j=0}^{i}\left(\int_{\mathbb{S}^{d}} f \Delta^{2 j} f d \sigma-\int_{\mathbb{S}^{d}} f \Delta^{2 j+1} f d \sigma\right)=\sum_{j=0}^{i} \int_{\mathbb{S}^{d}}\left(\left|\Delta^{j} f\right|^{2}+\left|\nabla \Delta^{j} f\right|^{2}\right) d \sigma
\end{aligned}
$$

where the comparability constants only depend on $d$ and $m$. The same argument applies in case that $m \in \mathbb{N}$ is even. Thus, we get the following well-known result.

Lemma 2.7. For $m=0,2,4,6,8, \ldots$ we have

$$
\begin{aligned}
\|f\|_{\mathbb{H}^{m}\left(\mathbb{S}^{d}\right)}^{2} \approx & \|f\|_{L^{2}\left(\mathbb{S}^{d}\right)}^{2}+\|\nabla f\|_{L^{2}\left(\mathbb{S}^{d}\right)}^{2}+\|\Delta f\|_{L^{2}\left(\mathbb{S}^{d}\right)}^{2}+\|\nabla \Delta f\|_{L^{2}\left(\mathbb{S}^{d}\right)}^{2} \\
& +\ldots+\left\|\Delta^{\frac{m-2}{2}} f\right\|_{L^{2}\left(\mathbb{S}^{d}\right)}^{2}+\left\|\nabla \Delta^{\frac{m-2}{2}} f\right\|_{L^{2}\left(\mathbb{S}^{d}\right)}^{2}+\left\|\Delta^{\frac{m}{2}} f\right\|_{L^{2}\left(\mathbb{S}^{d}\right)}^{2}
\end{aligned}
$$


and for $m=1,3,5,7,9, \ldots$ we have

$$
\begin{aligned}
\|f\|_{\mathbb{H}^{m}\left(\mathbb{S}^{d}\right)}^{2} \approx & \|f\|_{L^{2}\left(\mathbb{S}^{d}\right)}^{2}+\|\nabla f\|_{L^{2}\left(\mathbb{S}^{d}\right)}^{2}+\|\Delta f\|_{L^{2}\left(\mathbb{S}^{d}\right)}^{2}+\|\nabla \Delta f\|_{L^{2}\left(\mathbb{S}^{d}\right)}^{2} \\
& +\ldots+\left\|\nabla \Delta^{\frac{m-3}{2}} f\right\|_{L^{2}\left(\mathbb{S}^{d}\right)}^{2}+\left\|\Delta^{\frac{m-1}{2}} f\right\|_{L^{2}\left(\mathbb{S}^{d}\right)}^{2}+\left\|\nabla \Delta^{\frac{m-1}{2}} f\right\|_{L^{2}\left(\mathbb{S}^{d}\right)}^{2} .
\end{aligned}
$$

With the expressions of $\|\cdot\|_{\mathbb{H}^{m}\left(\mathbb{S}^{d}\right)}$ from Lemma 2.7 at hand, one can now take a new look to (2.6).

\section{Asymptotic estimates of Riesz energies}

This section focuses on asymptotic estimates of Riesz energies on the sphere. The main result, namely Corollary 3.7, is an estimate of the continuous Riesz energy of small discs centered at the discrete minimizers in terms of the minimal energy $\mathcal{E}_{s}(N)$ defined in (1.1), plus error terms. This, together with the asymptotic expansion of the minimal energy, will be a key tool in the next section to derive the estimates of the Sobolev discrepancy given in Theorem 1.5.

To prove Corollary 3.7, we treat the supercritical and subcritical regimes separately. In the first one, we essentially make use of the separation of the point minimizers, a decomposition of the sphere in dyadic annuli, and Gauss-Green formula (2.9). This is carried out in Lemma 3.2. Proposition 3.3, and Theorem 3.4 below. Since in the subcritical case the separation property is not known to hold, in Lemma 3.1 below we overcome this difficulty by making use of the fact that $R_{s}\left(\cdot, x_{0}\right)$ is superharmonic near $x_{0}$ when $0 \leq s<d-2$ for $d>2$, as (2.7) shows. We mention that the original argument of Wolff for the logarithmic kernel in $\mathbb{S}^{2}$ was already based on the use of superharmonicity.

Lemma 3.1. For $d>2$ and $0<s<d-2$ there exist $\delta, C>0$ depending only on $s$ and $d$, such that for every $a, b \in \mathbb{S}^{d}$,

$$
f_{D_{r}(a)} f_{D_{r}(b)} R_{s}(x, y) d \sigma(x) d \sigma(y) \leq R_{s}(a, b)+C r^{2},
$$

with, say, $0<r<\frac{\delta}{100}$.

For $d \geq 2$ there exist $C>0$ depending only on $d$ such that for every $a, b \in \mathbb{S}^{d}$,

$$
f_{D_{r}(a)} f_{D_{r}(b)} R_{0}(x, y) d \sigma(x) \leq R_{0}(a, b)+C r^{2}
$$

for all $0<r \leq 1$.

Proof. Let $a, b \in \mathbb{S}^{d}$ and $\delta>0$ small enough to be chosen later on. We take $r>0$ such that, say, $0<r<\frac{\delta}{100}$ and we split the argument into two cases,

$$
D_{r}(b) \subset D_{2 \delta}(a) \text { or } \quad D_{r}(b) \subset \mathbb{S}^{d} \backslash D_{\delta}(a) .
$$

Observe that from Lemma 2.5 lemma we get

$$
\Delta_{x} R_{s}(x, y)=s\left[\frac{(2 d-s-2)}{4}|x-y|^{2}-(d-s-2)\right] R_{s+2}(x, y) .
$$

Thus, there exist $\delta>0$ small enough depending on $s$ and $d$ such that for $|x-y|<10 \delta$

$$
\Delta_{x} R_{s}(x, y) \leq 0 .
$$


In the first case $D_{r}(b) \subset D_{2 \delta}(a)$, we get $D_{r}(a), D_{r}(b) \subset D_{2 \delta}(a)$ and, therefore,

$$
\Delta_{x} R_{s}(x, y) \leq 0, \quad \Delta_{y} R_{s}(x, y) \leq 0,
$$

for all $x \in D_{r}(a)$ and $y \in D_{r}(b)$. Then we get

$$
f_{D_{r}(b)} R_{s}(a, y) d \sigma(y) \leq R_{s}(a, b) \quad \text { and } \quad f_{D_{r}(a)} R_{s}(x, y) d \sigma(x) \leq R_{s}(a, y)
$$

for all $y \in D_{r}(b)$ and, therefore,

$$
f_{D_{r}(a)} f_{D_{r}(b)} R_{s}(x, y) d \sigma(x) d \sigma(y) \leq R_{s}(a, b) .
$$

In the second case $D_{r}(b) \subset \mathbb{S}^{d} \backslash D_{\delta}(a)$, we take the $\delta>0$ as in the first case. Observe that for $x \in D_{r}(a)$ and $y \in D_{r}(b)$ we have $|x-y|>\frac{\delta}{2}$ and therefore, from the explicit expressions above, $\Delta_{x} R_{s}(x, y)$ is bounded above by a constant $C_{s, d}>0$ depending only on $s$ and $d$. By a computation similar to one in Lemma 2.5 we have

$$
\Delta_{x}\left|x-x_{0}\right|^{2}=d\left(2-\left|x-x_{0}\right|^{2}\right)
$$

for all $x_{0} \in \mathbb{S}^{d}$, and therefore if $\left|x-x_{0}\right| \leq 1$ we have $\Delta_{x}\left|x-x_{0}\right|^{2} \geq d$. Then, taking $C=C_{s, d} / d$ and $x_{0}=a$, we get

$$
\Delta_{x}\left(R_{s}(x, y)-C|x-a|^{2}\right) \leq 0, \quad x \in D_{r}(a) .
$$

From this superharmonicity and the corresponding mean value inequality we obtain

$$
f_{D_{r}(a)} R_{s}(x, y) d \sigma(x)-C f_{D_{r}(a)}|x-a|^{2} d \sigma(x) \leq R_{s}(a, y)
$$

and, thus,

$$
f_{D_{r}(a)} R_{s}(x, y) d \sigma(x) \leq R_{s}(a, y)+C r^{2}
$$

Similarly, we get

$$
f_{D_{r}(b)} R_{s}(a, y) d \sigma(y)-C f_{D_{r}(b)}|y-b|^{2} d \sigma(y) \leq R_{s}(a, b),
$$

and

$$
f_{D_{r}(b)} R_{s}(a, y) d \sigma(y) \leq R_{s}(a, b)+C r^{2} .
$$

Combining (3.2) and (3.3) we finally obtain

$$
f_{D_{r}(a)} f_{D_{r}(b)} R_{s}(x, y) d \sigma(x) d \sigma(y) \leq R_{s}(a, b)+C r^{2},
$$

and the result follows together with (3.1).

In the logarithmic case we argue as above and take $a, b \in \mathbb{S}^{d}$ and $0<r \leq 1$. Then, in the distributional sense, for every $y \in D_{r}(b)$,

and

$$
\Delta_{x}\left(R_{0}(\cdot, y)-\frac{d-1}{2 d}|\cdot-a|^{2}\right) \leq 0, \quad \text { in } D_{r}(a),
$$

$$
\Delta_{y}\left(R_{0}(a, \cdot)-\frac{d-1}{2 d}|\cdot-b|^{2}\right) \leq 0, \quad \text { in } D_{r}(b) .
$$


It follows that

$$
f_{D_{r}(a)} f_{D_{r}(b)} R_{0}(x, y) d \sigma(x) d \sigma(y) \leq R_{0}(a, b)+\frac{d-1}{d} r^{2} .
$$

Lemma 3.2. Let $d>2,0<s<d, r_{0}>0$, and $x_{0}, x_{1} \in \mathbb{S}^{d}$ be such that $\left|x_{0}-x_{1}\right|>r_{0}$. Then,

$$
\begin{aligned}
R_{s}\left(x_{0}, x_{1}\right)= & \frac{d s(d-2-s)}{(d-2) C_{d} r_{0}^{d}} \int_{0}^{r_{0}} \int_{D_{r}\left(x_{0}\right)} r^{d-1}\left(R_{d-2}\left(x, x_{0}\right)-r^{2-d}\right) R_{s+2}\left(x, x_{1}\right) d \sigma(x) d r \\
& +\frac{d(d-s)(d-2-s)}{4(d-2) C_{d} r_{0}^{d}} \int_{0}^{r_{0}} \int_{D_{r}\left(x_{0}\right)} r^{d-1} R_{d-2}\left(x, x_{0}\right) R_{s}\left(x, x_{1}\right) d \sigma(x) d r \\
& +\frac{d s(2 d-2-s)}{4(d-2) C_{d} r_{0}^{d}} \int_{0}^{r_{0}} \int_{D_{r}\left(x_{0}\right)} r R_{s}\left(x, x_{1}\right) d \sigma(x) d r \\
& +\frac{d}{C_{d} r_{0}^{d}} \int_{D_{r_{0}}\left(x_{0}\right)}\left(1-\frac{1}{4}\left|x-x_{0}\right|^{2}\right) R_{s}\left(x, x_{1}\right) d \sigma(x),
\end{aligned}
$$

where $C_{d}=2 \pi^{d / 2} / \Gamma(d / 2)$.

Proof. Let $0<r<r_{0}$. Applying (2.9) with $\Omega=D_{r}\left(x_{0}\right)$ and $f(x)=R_{s}\left(x, x_{1}\right)$ we get

$$
\begin{aligned}
C_{d}(d-2) R_{s}\left(x_{0}, x_{1}\right)= & \int_{D_{r}\left(x_{0}\right)} R_{d-2}\left(x, x_{0}\right)\left(-\Delta+\frac{1}{4}(d-2) d\right) R_{s}\left(x, x_{1}\right) d \sigma(x) \\
& +\int_{\partial D_{r}\left(x_{0}\right)} R_{d-2}\left(x, x_{0}\right) \nabla R_{s}\left(x, x_{1}\right) \cdot \nu(x) d \sigma^{\prime}(x) \\
& -\int_{\partial D_{r}\left(x_{0}\right)} R_{s}\left(x, x_{1}\right) \nabla R_{d-2}\left(x, x_{0}\right) \cdot \nu(x) d \sigma^{\prime}(x) \\
= & : I_{1}(r)+I_{2}(r)+I_{3}(r),
\end{aligned}
$$

where, as before, $\sigma^{\prime}$ stands for the $(d-1)$-dimensional Hausdorff measure. The proof of (3.4) is based on multiplying (3.5) by $r^{d-1}$ and integrating over all $r \in\left(0, r_{0}\right)$. We deal with the three terms on the right hand side of (3.5) separately. On one hand, using (2.7) we get

$$
I_{1}(r)=(d-2-s) \int_{D_{r}\left(x_{0}\right)} R_{d-2}\left(x, x_{0}\right)\left(\frac{d-s}{4} R_{s}\left(x, x_{1}\right)+s R_{s+2}\left(x, x_{1}\right)\right) d \sigma(x) .
$$

Regarding $I_{2}(r)$, since $R_{d-2}\left(x, x_{0}\right)=r^{2-d}$ for all $x \in \partial D_{r}\left(x_{0}\right)$, the divergence theorem and (2.7) yield

$$
\begin{aligned}
I_{2}(r) & =r^{2-d} \int_{D_{r}\left(x_{0}\right)} \Delta R_{s}\left(x, x_{1}\right) d \sigma(x) \\
& =r^{2-d} s \int_{D_{r}\left(x_{0}\right)}\left(\frac{1}{4}(2 d-2-s) R_{s}\left(x, x_{1}\right)-(d-2-s) R_{s+2}\left(x, x_{1}\right)\right) d \sigma(x) .
\end{aligned}
$$


Finally, arguing as in (2.16) we deduce that

$$
I_{3}(r)=\frac{d-2}{r^{d-1}} \int_{\partial D_{r}\left(x_{0}\right)}\left(\frac{1+\left(x_{0} \cdot x\right)}{2}\right)^{1 / 2} R_{s}\left(x, x_{1}\right) d \sigma^{\prime}(x) .
$$

A combination of (3.8) and the smooth coarea formula, see [9, page 160], leads to

$$
\begin{aligned}
\int_{0}^{r_{0}} r^{d-1} I_{3}(r) d r & =(d-2) \int_{D_{r_{0}}\left(x_{0}\right)} \frac{1+\left(x_{0} \cdot x\right)}{2} R_{s}\left(x, x_{1}\right) d \sigma(x) \\
& =(d-2) \int_{D_{r_{0}}\left(x_{0}\right)}\left(1-\frac{\left|x-x_{0}\right|^{2}}{4}\right) R_{s}\left(x, x_{1}\right) d \sigma(x) .
\end{aligned}
$$

Therefore, if we multiply (3.5) by $r^{d-1}$ and we integrate over all $r \in\left(0, r_{0}\right)$, using (3.6)), (3.7) and (3.9) we finally get (3.4).

Proposition 3.3. Let $d>2$ and $0<s<d$. There exists $C>0$ only depending on $d$ and $s$ such that

$$
\left|R_{s}\left(x_{0}, x_{1}\right)-f_{D_{r_{0}}\left(x_{0}\right)} R_{s}\left(x, x_{1}\right) d \sigma(x)\right| \leq C\left(\psi_{d-2}(s) 2^{2 k}+1\right) 2^{k s} r_{0}^{2}
$$

for all $k \geq 0$, all $x_{0}, x_{1} \in \mathbb{S}^{d}$ with $\left|x_{0}-x_{1}\right| \geq 2^{-k}$ and all $0<r_{0} \leq 2^{-k-2}$, where we have set $\psi_{d-2}(s)=0$ if $s=d-2$ and $\psi_{d-2}(s)=1$ otherwise.

Proof. Thanks to (3.4), we can write

$$
\begin{aligned}
R_{s}\left(x_{0}, x_{1}\right)- & \frac{1}{\left|D_{r_{0}}\left(x_{0}\right)\right|} \int_{D_{r_{0}}\left(x_{0}\right)} R_{s}\left(x, x_{1}\right) d \sigma(x) \\
= & \frac{d s(d-2-s)}{(d-2) C_{d} r_{0}^{d}} \int_{0}^{r_{0}} \int_{D_{r}\left(x_{0}\right)} r^{d-1}\left(R_{d-2}\left(x, x_{0}\right)-r^{2-d}\right) R_{s+2}\left(x, x_{1}\right) d \sigma(x) d r \\
& +\frac{d(d-s)(d-2-s)}{4(d-2) C_{d} r_{0}^{d}} \int_{0}^{r_{0}} \int_{D_{r}\left(x_{0}\right)} r^{d-1} R_{d-2}\left(x, x_{0}\right) R_{s}\left(x, x_{1}\right) d \sigma(x) d r \\
& +\frac{d s(2 d-2-s)}{4(d-2) C_{d} r_{0}^{d}} \int_{0}^{r_{0}} \int_{D_{r}\left(x_{0}\right)} r R_{s}\left(x, x_{1}\right) d \sigma(x) d r \\
& -\frac{d}{4 C_{d} r_{0}^{d}} \int_{D_{r_{0}}\left(x_{0}\right)}\left|x-x_{0}\right|^{2} R_{s}\left(x, x_{1}\right) d \sigma(x) \\
& +\left(\frac{d}{C_{d} r_{0}^{d}}-\frac{1}{\left|D_{r_{0}}\left(x_{0}\right)\right|}\right) \int_{D_{r_{0}}\left(x_{0}\right)} R_{s}\left(x, x_{1}\right) d \sigma(x) \\
= & : S_{1}+S_{2}+S_{3}+S_{4}+S_{5} .
\end{aligned}
$$

We are going to estimate the terms $S_{1}, \ldots, S_{5}$ separately. However, all the estimates rely basically on the assumptions $\left|x_{0}-x_{1}\right| \geq 2^{-k}$ and $r_{0} \leq 2^{-k-2}$. On one hand, we easily see that

$$
\begin{aligned}
\left|S_{1}\right| & \leq C \psi_{d-2}(s) 2^{k(s+2)} r_{0}^{-d} \int_{0}^{r_{0}} \int_{D_{r}\left(x_{0}\right)} r^{d-1}\left(\left|x-x_{0}\right|^{2-d}-r^{2-d}\right) d \sigma(x) d r \\
& \leq C \psi_{d-2}(s) 2^{k(s+2)} r_{0}^{2} .
\end{aligned}
$$


Similarly,

$$
\left|S_{2}\right| \leq C \psi_{d-2}(s) 2^{k s} r_{0}^{2}, \quad\left|S_{3}\right| \leq C 2^{k s} r_{0}^{2} \quad \text { and } \quad\left|S_{4}\right| \leq C 2^{k s} r_{0}^{2}
$$

Finally, by taking local chards in $\mathbb{S}^{d}$, one can show that ||$D_{r}(x)\left|-C_{d} r^{d} / d\right| \leq C r^{d+2}$ for all $0 \leq r \leq 2$ and all $x \in \mathbb{S}^{d}$. Hence,

$$
\left|S_{5}\right| \leq C r_{0}^{-2 d}|| D_{r}(x)\left|-C_{d} r^{d} / d\right| \int_{D_{r_{0}}\left(x_{0}\right)} R_{s}\left(x, x_{1}\right) d \sigma(x) \leq C 2^{k s} r_{0}^{2} .
$$

Plugging (3.12), (3.13) and (3.14) in (3.11), we obtain (3.10), as desired.

Theorem 3.4. Let $d>2,0<s<d$, and $\rho>0$. There exists $C>0$ only depending on $d, s$ and $\rho$ such that

$$
\frac{1}{N^{2}} \sum_{i \neq j}\left|R_{s}\left(x_{i}, x_{j}\right)-f_{D_{j}} f_{D_{i}} R_{s}(x, y) d \sigma(x) d \sigma(y)\right| \leq C \epsilon^{2}\left(N^{-\frac{2}{d}}+N^{-1+\frac{s}{d}}\right)
$$

for all $0<\epsilon \leq \rho / 8$, all $N \in \mathbb{N}$ and every sequence of points $\left\{x_{j}\right\}_{j=1, \ldots, N} \subset \mathbb{S}^{d}$ such that $\left|x_{i}-x_{j}\right| \geq \rho N^{-1 / d}$ for all $i \neq j$, where we have set $D_{j}=D_{\epsilon N^{-1 / d}}\left(x_{j}\right)$ for $j=1, \ldots, N$.

Proof. First of all, note that $D_{i} \cap D_{j}=\emptyset$ for all $i \neq j$ since $0<\epsilon \leq \rho / 8$ and $\left|x_{i}-x_{j}\right| \geq \rho N^{-1 / d}$. Therefore, the left hand side of (3.15) is well defined and finite for all $s \geq 0$.

Given $i \neq j$, since $\rho N^{-1 / d} \leq\left|x_{i}-x_{j}\right| \leq 2$, there exists some integer $k \geq 0$ such that

$$
\rho N^{-1 / d} / 2 \leq 2^{-k} \leq\left|x_{i}-x_{j}\right| \leq 2^{-k+1}
$$

Then, using the triangle inequality and (3.10) we can estimate

$$
\begin{aligned}
&\left|R_{s}\left(x_{i}, x_{j}\right)-f_{D_{j}} f_{D_{i}} R_{s}(x, y) d \sigma(x) d \sigma(y)\right| \\
& \leq\left|R_{s}\left(x_{i}, x_{j}\right)-f_{D_{j}} R_{s}\left(x_{i}, y\right) d \sigma(y)\right| \\
& \quad+f_{D_{j}}\left|R_{s}\left(x_{i}, y\right)-f_{D_{i}} R_{s}(x, y) d \sigma(x)\right| d \sigma(y) \\
& \leq C\left(\psi_{d-2}(s) 2^{2 k}+1\right) 2^{k s} \epsilon^{2} N^{-2 / d} .
\end{aligned}
$$

In addition, due to the constraint $\left|x_{i}-x_{j}\right| \geq \rho N^{-1 / d}$ for all $i \neq j$, it is not hard to show that there exists $C>0$ only depending on $d$ such that, for every $i$ and $k$,

$$
\#\left\{j: 2^{-k} \leq\left|x_{i}-x_{j}\right| \leq 2^{-k+1}\right\} \leq C 2^{-k d} \rho^{-d} N .
$$


Therefore, a combination of (3.16), (3.17) and (3.18) leads to

$$
\begin{aligned}
\sum_{i \neq j} \mid & R_{s}\left(x_{i}, x_{j}\right)-f_{D_{j}} f_{D_{i}} R_{s}(x, y) d \sigma(x) d \sigma(y) \mid \\
& \leq C \sum_{1 \leq i \leq N} \sum_{0 \leq k \leq \log _{2}\left(\frac{2 N^{1 / d}}{\rho}\right)} \sum_{j: 2^{-k} \leq\left|x_{i}-x_{j}\right| \leq 2^{-k+1}}\left(\psi_{d-2}(s) 2^{2 k}+1\right) 2^{k s} \epsilon^{2} N^{-2 / d} \\
& \leq C \rho^{-d} \epsilon^{2} N^{2-2 / d} \sum_{0 \leq k \leq \log _{2}\left(\frac{2 N^{1 / d}}{\rho}\right)} 2^{-k(d-s)}\left(\psi_{d-2}(s) 2^{2 k}+1\right) .
\end{aligned}
$$

Recall that if $s=d-2$ then $\psi_{d-2}(s)=0$, thus from (3.19) we obtain in this case

$$
\sum_{i \neq j}\left|R_{d-2}\left(x_{i}, x_{j}\right)-f_{D_{j}} f_{D_{i}} R_{d-2}(x, y) d \sigma(x) d \sigma(y)\right| \leq C \epsilon^{2} N^{2-2 / d}
$$

for some $C>0$ only depending on $d, s$ and $\rho$. On the other hand, if $s \neq d-2$ then $\psi_{d-2}(s)=1$, and from (3.19) we get

$$
\begin{aligned}
\sum_{i \neq j} \mid R_{s}\left(x_{i}, x_{j}\right) & -f_{D_{j}} f_{D_{i}} R_{s}(x, y) d \sigma(x) d \sigma(y) \mid \\
& \leq C \rho^{-d} \epsilon^{2} N^{2-2 / d} \sum_{0 \leq k \leq \log _{2}\left(\frac{2 N^{1 / d}}{\rho}\right)} 2^{-k(d-2-s)} \\
& \leq C \epsilon^{2} N^{2-2 / d}\left(1+N^{(s-(d-2)) / d}\right)=C \epsilon^{2}\left(N^{2-2 / d}+N^{1+s / d}\right)
\end{aligned}
$$

for some $C>0$ only depending on $d, s$ and $\rho$, as before. In any case, (3.15) follows directly from (3.20) and (3.21).

Remark 3.5. The estimate (3.15) may not seem sharp but, as far as one bases it on a pointwise estimate of the factor inside the sum independently of $i$, in the spirit of (3.19), one cannot expect anything better than (3.15). This is essentially because the estimate in (3.10) is sharp for points in the sphere satisfying $2^{-k} \leq\left|x_{i}-x_{j}\right| \leq 2^{-k+1}$. That is to say, there exists $C>0$ such that

$$
C^{-1} 2^{k(s+2)} r_{0}^{2} \leq\left|R_{s}\left(x_{0}, x_{1}\right)-f_{D_{r_{0}}\left(x_{0}\right)} R_{s}\left(x, x_{1}\right) d \sigma(x)\right| \leq C 2^{k(s+2)} r_{0}^{2}
$$

whenever $s \neq d-2$ and $2^{-k} \leq\left|x_{0}-x_{1}\right| \leq 2^{-k+1}$, where $x_{0}, x_{1}$ and $r_{0}$ are as in Proposition 3.3. To see this simply note that $\left|S_{1}\right|$ in (3.11), which is comparable to $2^{k(s+2)} r_{0}^{2}$ and has a positive integrand, is the dominant term in the decomposition given in (3.11) as $k \rightarrow+\infty$. Thus, all the other terms $S_{j}$ can be absorbed by $S_{1}$ for $k$ big enough, and everything is comparable for $k$ small. This reasoning gives the lower bound in (3.22).

Remark 3.6. It is not hard to extend Theorem 3.4 to the more general case $d \geq 2$ and $0 \leq s<d$ by a suitable modification of Lemma 3.2 and Proposition 3.3 using the corresponding identities from Lemma 2.5, for example (2.11) instead of (2.9) when $d=2$, or (2.10) instead of (2.7) when $s=0$. We omit the details for the sake of shortness. 
Corollary 3.7. Given $0 \leq s<d$, let $\left\{x_{i}\right\}_{i=1}^{N}$ be an $N$ point set of minimizers of the Riesz s-energy. Then, there exist $\epsilon_{0}=\epsilon_{0}(s, d)>0$ such that, if $0<\epsilon<\epsilon_{0}$ and $D_{j}=D_{\epsilon N^{-1 / d}}\left(x_{j}\right)$ for $j=1, \ldots, N$,

$$
\frac{1}{N^{2}} \sum_{i \neq j} f_{D_{j}} f_{D_{i}} R_{s}(x, y) d \sigma(x) d \sigma(y) \leq \frac{\mathcal{E}_{s}(N)}{N^{2}}+C \epsilon^{2}\left(N^{-\frac{2}{d}}+N^{-1+\frac{s}{d}}\right),
$$

for some constant $C>0$ depending only on $d$ and $s$.

Proof. If $d \geq 2$ and $d-2 \leq s<d$ the minimizers are separated $\left|x_{i}-x_{j}\right| \geq c N^{-1 / d}$, and by taking $\epsilon_{0}>0$ small enough according to the separation the result follows from Theorem 3.4 and Remark 3.6. For $d>2$ and $0 \leq s<d-2$ we apply Lemma 3.1 to spherical caps of radius $\epsilon N^{-1 / d}$ for $\epsilon>0$ small enough.

\section{Estimates of the Sobolev discrepancy}

In this section we derive the estimates of the Sobolev discrepancy stated in Theorem 1.5, which are sharp for the range $d-2 \leq s<d$. The first result, that can be seen as a sort of Stolarky's invariance principle, generalizes Wolff's result on the Sobolev discrepancy for $\mathbb{S}^{2}$ and $s=0$.

Lemma 4.1. Let $D_{1}, \ldots, D_{N}$ be spherical caps in $\mathbb{S}^{d}$ of the same radius $r>0$. Consider the measures

$$
\mu_{i}=\frac{\chi_{D_{i}}}{\sigma\left(D_{i}\right)} \sigma, \quad \mu=\frac{1}{N} \sum_{i=1}^{N} \mu_{i}-\frac{\sigma}{\omega_{d}}
$$

In particular, $\mu\left(\mathbb{S}^{d}\right)=0$. Let $K(x, y)=K(|x-y|)$ be a rotation invariant integrable kernel. Then,

$$
\begin{aligned}
\frac{1}{N^{2}} \sum_{i \neq j} \int_{\mathbb{S}^{d}} \int_{\mathbb{S}^{d}} K(x, y) d \mu_{i}(x) d \mu_{j}(y)= & \frac{1}{\omega_{d}^{2}} \int_{\mathbb{S}^{d}} \int_{\mathbb{S}^{d}} K(x, y) d \sigma(x) d \sigma(y) \\
& +\int_{\mathbb{S}^{d}} \int_{\mathbb{S}^{d}} K(x, y) d \mu(x) d \mu(y) \\
& -\frac{1}{N} f_{D} f_{D} K(x, y) d \sigma(x) d \sigma(y),
\end{aligned}
$$

where $D$ is a spherical cap of radius $r$ centered at the north pole.

Proof. Writing the measure $\mu$ in terms of its summands we have

$$
\begin{aligned}
\int_{\mathbb{S}^{d}} \int_{\mathbb{S}^{d}} K(x, y) d \mu(x) d \mu(y)= & \frac{1}{N^{2}} \sum_{i, j} \int_{\mathbb{S}^{d}} \int_{\mathbb{S}^{d}} K(x, y) d \mu_{i}(x) d \mu_{j}(y) \\
& -\frac{2}{N \omega_{d}} \sum_{i} \int_{\mathbb{S}^{d}} \int_{\mathbb{S}^{d}} K(x, y) d \mu_{i}(x) d \sigma(y) \\
& +\frac{1}{\omega_{d}^{2}} \int_{\mathbb{S}^{d}} \int_{\mathbb{S}^{d}} K(x, y) d \sigma(x) d \sigma(y) .
\end{aligned}
$$


Now, observing that

$$
2\left(\frac{1}{N} \sum_{i} d \mu_{i}(x)\right) \frac{d \sigma(y)}{\omega_{d}}-\frac{d \sigma(x) d \sigma(y)}{\omega_{d}^{2}}=\frac{d \sigma(x) d \sigma(y)}{\omega_{d}^{2}}+2 \frac{d \mu(x) d \sigma(y)}{\omega_{d}},
$$

we obtain

$$
\begin{aligned}
\int_{\mathbb{S}^{d}} \int_{\mathbb{S}^{d}} K(x, y) d \mu(x) d \mu(y)= & \frac{1}{N^{2}} \sum_{i \neq j} \int_{\mathbb{S}^{d}} \int_{\mathbb{S}^{d}} K(x, y) d \mu_{i}(x) d \mu_{j}(y) \\
& +\frac{1}{N^{2}} \sum_{i} \int_{\mathbb{S}^{d}} \int_{\mathbb{S}^{d}} K(x, y) d \mu_{i}(x) d \mu_{i}(y) \\
& -\frac{1}{\omega_{d}^{2}} \int_{\mathbb{S}^{d}} \int_{\mathbb{S}^{d}} K(x, y) d \sigma(x) d \sigma(y) \\
& -\frac{2}{\omega_{d}} \int_{\mathbb{S}^{d}} \int_{\mathbb{S}^{d}} K(x, y) d \mu(x) d \sigma(y) .
\end{aligned}
$$

The last integral vanishes because $\mu\left(\mathbb{S}^{d}\right)=0$ and $K(x, y)$ is rotation invariant. Moreover, by rotation invariance, the integrals

$$
\int_{\mathbb{S}^{d}} \int_{\mathbb{S}^{d}} K(x, y) d \mu_{i}(x) d \mu_{i}(y)
$$

are all equal and independent of the center of the spherical cap.

Proposition 4.2. Let $D_{1}, \ldots, D_{N}$ be spherical caps in $\mathbb{S}^{d}$ of the same radius $\epsilon N^{-1 / d}$. Consider the measures

$$
\mu_{i}=\frac{\chi_{D_{i}}}{\sigma\left(D_{i}\right)} \sigma, \quad \mu=\frac{1}{N} \sum_{i=1}^{N} \mu_{i}-\frac{\sigma}{\omega_{d}}
$$

Then,

$$
E_{s}(\widetilde{\sigma})+E_{s}(\mu)-\frac{1}{N^{2}} \sum_{i \neq j} \int_{\mathbb{S}^{d}} \int_{\mathbb{S}^{d}} R_{s}(x, y) d \mu_{i}(x) d \mu_{j}(y) \approx \epsilon^{-s} N^{-1+\frac{s}{d}}
$$

for $0<s<d$, where $\widetilde{\sigma}=\sigma / \omega_{d}$ is the normalized surface measure in $\mathbb{S}^{d}$. If $s=0$, then

$$
\frac{1}{N^{2}} \sum_{i \neq j} \int_{\mathbb{S}^{d}} \int_{\mathbb{S}^{d}} R_{0}(x, y) d \mu_{i}(x) d \mu_{j}(y)=E_{0}(\widetilde{\sigma})+E_{0}(\mu)-\frac{1}{d} \frac{\log N}{N}+O\left(N^{-1}\right) .
$$

Proof. For $0<s<d$ we take $K=R_{s}$ in Lemma 4.1 to deduce that

$$
\frac{1}{N^{2}} \sum_{i \neq j} \int_{\mathbb{S}^{d}} \int_{\mathbb{S}^{d}} R_{s}(x, y) d \mu_{i}(x) d \mu_{j}(y)=E_{s}(\widetilde{\sigma})+E_{s}(\mu)-\frac{1}{N} f_{D} f_{D} R_{s}(x, y) d \sigma(x) d \sigma(y),
$$

where $D$ denotes a spherical cap of radius $\epsilon N^{-1 / d}$ centered at the north pole $n=$ $(0, \ldots, 0,1)$. To estimate this last integral we use normal coordinates around the north pole, say,

$$
\Phi(x)= \begin{cases}(0, \ldots, 0,1), & \text { if } x=0 \\ \left(\frac{x}{|x|} \sin |x|, \cos |x|\right), & \text { if } x \neq 0\end{cases}
$$


for $x \in \mathbb{R}^{d}$ with $|x| \leq \pi$. For every $r>0$, we have

$$
\begin{aligned}
& \int_{D(n, r)} \int_{D(n, r)} R_{s}(x, y) d \sigma(x) d \sigma(y) \\
& \quad=\int_{B\left(0, \arccos \left(1-\frac{r^{2}}{2}\right)\right)} \int_{B\left(0, \arccos \left(1-\frac{r^{2}}{2}\right)\right)} R_{s}(\Phi(x), \Phi(y))\left(\frac{\sin |x|}{|x|}\right)^{d-1}\left(\frac{\sin |y|}{|y|}\right)^{d-1} d x d y,
\end{aligned}
$$

where $B(a, r)$ is the ball in $\mathbb{R}^{d}$ of center $a \in \mathbb{R}^{d}$ and radius $r>0$. As there exist constants $C, c>0$ such that $c|x-y| \leq|\Phi(x)-\Phi(y)| \leq C|x-y|$ and $1 / 2 \leq \sin t / t \leq 1$ for all $|t| \leq \pi / 2$, we deduce that

$$
\int_{D(n, r)} \int_{D(n, r)} R_{s}(x, y) d \sigma(x) d \sigma(y) \approx \int_{B\left(0, \arccos \left(1-\frac{r^{2}}{2}\right)\right)} \int_{B\left(0, \arccos \left(1-\frac{r^{2}}{2}\right)\right)} R_{s}(x, y) d x d y .
$$

Finally, it is easy to check that, for $0<s<d$,

$$
f_{B(0, r)} f_{B(0, r)} R_{s}(x-y) d x d y=r^{-s} f_{B(0,1)} f_{B(0,1)} R_{s}(x-y) d x d y
$$

and

$$
f_{B(0, r)} f_{B(0, r)} R_{0}(x-y) d x d y=\log \frac{1}{r}+f_{B(0,1)} f_{B(0,1)} R_{s}(x-y) d x d y,
$$

where we used that $|B(0,1)|=\omega_{d-1} / d$. Then, since $\frac{1}{2}|B(0, r)| \leq \sigma(D(n, r)) \leq|B(0, r)|$, we conclude that, for $0<s<d$,

$$
C \frac{N^{s / d}}{\epsilon^{s}} \leq f_{D} f_{D} R_{s}(x, y) d \sigma(x) d \sigma(y) \leq C^{-1} \frac{N^{s / d}}{\epsilon^{s}}
$$

for some constant $C>0$ depending only on $s$ and $d$. In the case $s=0$, we get that

$$
f_{D} f_{D} R_{0}(x, y) d \sigma(x) d \sigma(y)=\frac{1}{d} \log N+C+o(1), \quad N \rightarrow+\infty,
$$

where $C \in \mathbb{R}$ depends only on $d$.

Proof of Theorem 1.5. We first show that the Sobolev discrepancy of the measures associated to any set of $N$ points is bounded below. Given $X_{N}=\left\{x_{1}, \ldots, x_{N}\right\} \subset \mathbb{S}^{d}$ and $\epsilon>0$ define the measures

$$
\mu_{i}=\frac{\chi_{D_{i}}}{\sigma\left(D_{i}\right)} \sigma, \quad \mu_{X_{N}, \epsilon}=\frac{1}{N} \sum_{i=1}^{N} \mu_{i}-\frac{\sigma}{\omega_{d}}
$$

where $D_{j}=D_{\epsilon N^{-1 / d}}\left(x_{j}\right)$. Since the $\mu_{i}$ are probability measures, we have

$$
\begin{aligned}
\mathcal{E}_{s}(N) & \leq \int_{\mathbb{S}^{d}} \ldots \int_{\mathbb{S}^{d}}\left(\sum_{i \neq j} R_{s}\left(x_{i}, x_{j}\right)\right) d \mu_{1}\left(x_{1}\right) \ldots d \mu_{N}\left(x_{N}\right) \\
& =\sum_{i \neq j} \iint R_{s}(x, y) d \mu_{i}(x) d \mu_{j}(y) .
\end{aligned}
$$


Combining (4.2), the lower estimate for the minimal energy in (1.2), (1.3), Proposition 4.2, and Lemma 2.4 we get the lower bound

$$
D_{s, d}^{\epsilon}\left(X_{N}\right)^{2} \geq\left(-c+C \epsilon^{-s}\right) N^{-1+\frac{s}{d}}
$$

where $c>0$ is the constant in (1.2) and $C>0$ is the constant in (4.1). Observe that the bound in (4.3) is not trivial for $\epsilon$ small enough.

For the upper bound we use again Proposition 4.2, Corollary 3.7, the upper estimates for the minimal energy in (1.2), (1.3) and Lemma 2.4. We obtain that, for all $0<\epsilon<$ $\epsilon_{0}(s, d)$ and $0<s<d$,

$$
D_{s, d}^{\epsilon}\left(X_{N}\right)^{2} \leq C \epsilon^{2} N^{-2 / d}+\left(C+C \epsilon^{-s}+C \epsilon^{2}\right) N^{-1+\frac{s}{d}}
$$

and for $s=0$ and $d>2$

$$
D_{0, d}^{\epsilon}\left(X_{N}\right)^{2} \leq\left(C \epsilon^{2}+C\right) N^{-2 / d}
$$

Remark 4.3. In the manuscript [29], Wolff uses the asymptotic expansion of the discrete minimal energy

$$
\sum_{i \neq j} \log \frac{1}{\left|x_{i}-x_{j}\right|}=\frac{N^{2}}{(4 \pi)^{2}} \int_{\mathbb{S}^{2}} \int_{\mathbb{S}^{2}} \log \frac{1}{|x-y|} d \sigma(x) d \sigma(y)-\frac{N}{2} \log N+O(N),
$$

as $N \rightarrow+\infty$, which it seems it was not known at that time. In fact, in the manuscript Wolff mentions that he borrows the direction $\geq$ in (4.4) from Elkies [20, page 150] and proves the other, hence the manuscript must precede Wagner's bound [28]. This agrees with the information we have from Eremenko about Wolff giving him the manuscript around 1992. We will schetch now the main ideas in the manuscript to prove the inequality $\leq$ in (4.4). First, Wolff constructs area regular partitions on the sphere with pieces satisfying the Poincaré inequality. He calls a set of points allowable if it is defined by taking one point on each of these pieces of the area regular partition. Using Poincaré inequality he proves that allowable sets have minimal Sobolev discrepancy, i.e., of order $N^{-1}$. Finally, by using the case $s=0$ and $d=2$ of the decomposition in Lemma 4.1 and Lemma 3.1, he proves that allowable sets (and therefore Fekete points too) have logarithmic energy bounded above by the right hand side of (4.4).

\section{From the Sobolev Discrepancy to the SPHerical CAP Discrepancy}

The final ingredient to prove Theorem 1.1 is to estimate the spherical cap discrepancy using the bounds on the Sobolev discrepancy and a suitable test function. The main difficulty compared to Wolff's case [29] is that we need the following result on interpolation to be able to estimate the test function.

Lemma 5.1. . If $0 \leq s<d$, there exists $C>0$ only depending on $d$ and $s$ such that

$$
\left|\int f g d \sigma\right| \leq C\|f\|_{\mathbb{H}[(d-s) / 2]+1\left(\mathbb{S}^{d}\right)}^{\theta}\|f\|_{\mathbb{H}[(d-s) / 2]\left(\mathbb{S}^{d}\right)}^{1-\theta}\|g\|_{\mathbb{H}^{\frac{s-d}{2}}\left(\mathbb{S}^{d}\right)}
$$

for all $f \in \mathcal{C}^{\infty}\left(\mathbb{S}^{d}\right)$ and $g \in L^{2}\left(\mathbb{S}^{d}\right)$, where $\theta=(d-s) / 2-[(d-s) / 2] \in[0,1)$. 
Proof. We decompose $f$ and $g$ in spherical harmonics as

$$
f=\sum_{\ell, k} f_{\ell, k} Y_{\ell, k}, \quad g=\sum_{\ell, k} g_{\ell, k} Y_{\ell, k},
$$

where $f_{\ell, k}=\int f Y_{\ell, k} d \sigma$ and $g_{\ell, k}=\int g Y_{\ell, k} d \sigma$. Then, using Cauchy-Schwarz inequality, (2.3) and (2.1), we easily get

$$
\begin{aligned}
\left|\int f g d \sigma\right|^{2} & \leq\left(\sum_{\ell, k}\left|f_{\ell, k}\right|\left|g_{\ell, k}\right|\right)^{2} \lesssim \sum_{\ell, k}\left(1+\ell^{d-s}\right)\left|f_{\ell, k}\right|^{2} \sum_{\ell, k} A_{\ell, s}\left|g_{\ell, k}\right|^{2} \\
& =\|g\|_{\mathbb{H}^{\frac{s-d}{2}}\left(\mathbb{S}^{d}\right)}^{2} \sum_{\ell, k}\left(1+\ell^{d-s}\right)\left|f_{\ell, k}\right|^{2} .
\end{aligned}
$$

For a general $s$, the last sum above may correspond to a Sobolev norm of noninteger order. We are going to estimate it, by interpolation, in terms of Sobolev norms of integer order. Let $m \in \mathbb{N} \cup\{0\}$ be such that $s \in[d-2(m+1), d-2 m)$ and set $\delta=(d-s) / 2-m$, hence $0<\delta \leq 1$ and $d-s=2(m+\delta)$.

Assume first that $\delta=1$, thus $d-s=2(m+1)$ and

$$
\sum_{\ell, k}\left(1+\ell^{d-s}\right)\left|f_{\ell, k}\right|^{2}=\sum_{\ell, k}\left(1+\ell^{2(m+1)}\right)\left|f_{\ell, k}\right|^{2} \approx\|f\|_{\mathbb{H}^{m+1}\left(\mathbb{S}^{d}\right)}^{2}=\|f\|_{\mathbb{H}^{(d-s) / 2}\left(\mathbb{S}^{d}\right)}^{2} .
$$

With this at hand, (5.2) leads to (5.1) when $\theta=0$.

Assume now that $0<\delta<1$. Using that $\ell \geq 0$, we can estimate

$$
1+\ell^{d-s}=1+\ell^{2(m+\delta)} \leq\left(1+\ell^{m}\right)^{2}\left(1+\ell^{2 \delta}\right) .
$$

Since $1<1 / \delta<+\infty$, a combination of (5.3) with Hölder inequality yields

$$
\begin{aligned}
\sum_{\ell, k}\left(1+\ell^{d-s}\right)\left|f_{\ell, k}\right|^{2} & \leq \sum_{\ell, k}\left(\left(1+\ell^{m}\right)\left|f_{\ell, k}\right|\right)^{2 \delta}\left(1+\ell^{2 \delta}\right)\left(\left(1+\ell^{m}\right)\left|f_{\ell, k}\right|\right)^{2-2 \delta} \\
& \leq\left(\sum_{\ell, k}\left(\left(1+\ell^{m}\right)\left|f_{\ell, k}\right|\right)^{2}\left(1+\ell^{2 \delta}\right)^{\frac{1}{\delta}}\right)^{\delta}\left(\sum_{\ell, k}\left(\left(1+\ell^{m}\right)\left|f_{\ell, k}\right|\right)^{2}\right)^{1-\delta} \\
& \lesssim\left(\sum_{\ell, k}\left(1+\ell^{2(m+1)}\right)\left|f_{\ell, k}\right|^{2}\right)^{\delta}\left(\sum_{\ell, k}\left(1+\ell^{2 m}\right)\left|f_{\ell, k}\right|^{2}\right)^{1-\delta} \\
& \approx\|f\|_{\mathbb{H}^{m+1}\left(\mathbb{S}^{d}\right)}^{2 \delta}\|f\|_{\mathbb{H}^{m}\left(\mathbb{S}^{d}\right)^{2-2 \delta}}
\end{aligned}
$$

The fact that $\delta<1$ leads to $m=[(d-s) / 2]$, and therefore we conclude that

$$
\left(\sum_{\ell, k}\left(1+\ell^{d-s}\right)\left|f_{\ell, k}\right|^{2}\right)^{1 / 2} \lesssim\|f\|_{\mathbb{H}^{[(d-s) / 2]+1}\left(\mathbb{S}^{d}\right)}^{(d-s) / 2-[(d-s) / 2]}\|f\|_{\mathbb{H}[(d-s) / 2]\left(\mathbb{S}^{d}\right)}^{1+[(d-s) / 2]-(d-s) / 2}
$$

This, together with (5.2), proves (5.1) when $0<\theta<1$.

Finally, the following proposition combined with Theorem 1.5 proves Theorem 1.1.

Proposition 5.2. Given $0 \leq s<d, \epsilon_{0}>0$ and $C_{1}>0$, there exists $C_{2}>0$ only depending on $d, s, \epsilon_{0}$, and $C_{1}$ such that, for every set $X_{N}=\left\{x_{1}, \ldots, x_{N}\right\} \subset \mathbb{S}^{d}$ with Sobolev discrepancy

$$
D_{s, d}^{\epsilon_{0}}\left(X_{N}\right) \leq C_{1}\left(N^{-\frac{1}{d}}+N^{-\frac{1}{2}+\frac{s}{2 d}}\right)
$$


the spherical cap discrepancy of $X_{N}$ satisfies

$$
\sup _{D}\left|\frac{\#\left(X_{N} \cap D\right)}{N}-\frac{\sigma(D)}{\sigma\left(\mathbb{S}^{d}\right)}\right| \leq C_{2}\left(\chi_{[0, d-2]}(s) N^{-\frac{2}{d(d-s+1)}}+\chi_{(d-2, d)}(s) N^{-\frac{2(d-s)}{d(d-s+4)}}\right)
$$

where the supremum in the left hand side of (5.5) runs over all spherical caps $D \subset \mathbb{S}^{d}$.

Proof. Let $D=D_{r}(z)$ for $z \in \mathbb{S}^{d}$ and $r>0$. Given $0<\epsilon<r / 2$ let $f_{\epsilon}^{ \pm} \in \mathcal{C}^{\infty}\left(\mathbb{S}^{d}\right)$ be such that $0 \leq f_{\epsilon}^{ \pm} \leq 1$,

$$
f_{\epsilon}^{+}(x)=\left\{\begin{array}{ll}
1, & \text { if }|x-z|<r+\epsilon, \\
0, & \text { if }|x-z|>r+2 \epsilon,
\end{array} \quad f_{\epsilon}^{-}(x)= \begin{cases}1, & \text { if }|x-z|<r-2 \epsilon \\
0, & \text { if }|x-z|>r-\epsilon\end{cases}\right.
$$

Note that

$$
\sigma(D)-C \epsilon \leq \int f_{\epsilon}^{-} d \sigma \leq \int f_{\epsilon}^{+} d \sigma \leq \sigma(D)+C \epsilon
$$

for some constant $C>0$ only depending on $d$. It is not hard to see that $f_{\epsilon}^{ \pm}$can be taken in such a way that

$$
\left\|f_{\epsilon}^{ \pm}\right\|_{\mathbb{H}^{m}\left(\mathbb{S}^{d}\right)} \leq C\left(1+\epsilon^{-m+\frac{1}{2}}\right) \quad \text { for all } m \in \mathbb{N} \cup\{0\}
$$

For example, take a smooth function $\phi: \mathbb{R} \rightarrow \mathbb{R}$ such that $\chi_{(-\infty, 0]} \leq \phi \leq \chi_{(-\infty, 1)}$ and set $f_{\epsilon}^{+}(x)=\phi\left(\epsilon^{-1}(|x-z|-r-\epsilon)\right)$ and $\left.f_{\epsilon}^{-}(x)=\phi\left(\epsilon^{-1}(|x-z|-r+2 \epsilon)\right)\right)$. We leave the details of checking (15.7) for the reader.

Observe that if $\epsilon>\epsilon_{0} N^{-1 / d}$ then $f_{\epsilon}^{+} \equiv 1$ in $D_{\epsilon_{0} N^{-1 / d}}\left(x_{j}\right)$ for all $x_{j} \in D$. Recall also form Definition 1.3 that $\mu_{X_{N}, \epsilon_{0}}=h \sigma$ with

$$
h=\frac{1}{N} \sum_{j=1}^{N} \frac{\chi_{D_{j}}}{\sigma\left(D_{j}\right)}-\frac{1}{\sigma\left(\mathbb{S}^{d}\right)}, \quad D_{j}=D_{\epsilon_{0} N^{-1 / d}}\left(x_{j}\right) \text { for all } j=1, \ldots, N .
$$

Therefore,

$$
\frac{\#\left(D \cap X_{N}\right)}{N} \leq \frac{1}{N} \sum_{j=1}^{N} \frac{1}{\sigma\left(D_{j}\right)} \int_{D_{j}} f_{\epsilon}^{+} d \sigma=\int f_{\epsilon}^{+} d \mu_{X_{N}, \epsilon_{0}}+\frac{1}{\sigma\left(\mathbb{S}^{d}\right)} \int f_{\epsilon}^{+} d \sigma
$$

Similarly, since $f_{\epsilon}^{-} \leq 1$ and it is supported on the spherical cap or radius $r-\epsilon$ centered at $z$, we deduce that

$$
\frac{\#\left(D \cap X_{N}\right)}{N} \geq \frac{1}{N} \sum_{j=1}^{N} \frac{1}{\sigma\left(D_{j}\right)} \int_{D_{j}} f_{\epsilon}^{-} d \sigma=\int f_{\epsilon}^{-} d \mu_{X_{N}, \epsilon_{0}}+\frac{1}{\sigma\left(\mathbb{S}^{d}\right)} \int f_{\epsilon}^{-} d \sigma
$$


We have all the ingredients to prove (5.5). On one hand, if we first combine (5.8) and (5.6), and then we use that $\mu_{X_{N}, \epsilon_{0}}=h \sigma$, (5.1), (5.7) and (5.4), we get

$$
\begin{aligned}
& \frac{\#\left(D \cap X_{N}\right)}{N}-\frac{\sigma(D)}{\sigma\left(\mathbb{S}^{d}\right)} \leq \int f_{\epsilon}^{+} d \mu_{X_{N}, \epsilon_{0}}+C \epsilon=\int f_{\epsilon}^{+} h d \sigma+C \epsilon \\
& \leq C\left\|f_{\epsilon}^{+}\right\|_{\mathbb{H}[(d-s) / 2]+1\left(\mathbb{S}^{d}\right)}^{(d-s) / 2]}\left\|f_{\epsilon}^{+}\right\|_{\left.\mathbb{H}^{[}(d-s) / 2\right]\left(\mathbb{S}^{d}\right)}^{1-(d-s) / 2+[(d-s) / 2]}\|h\|_{\mathbb{H}(s-d) / 2\left(\mathbb{S}^{d}\right)}+C \epsilon \\
& \leq C\left(1+\epsilon^{-\left[\frac{d-s}{2}\right]-\frac{1}{2}}\right)^{\frac{d-s}{2}-\left[\frac{d-s}{2}\right]}\left(1+\epsilon^{-\left[\frac{d-s}{2}\right]+\frac{1}{2}}\right)^{1-\frac{d-s}{2}+\left[\frac{d-s}{2}\right]} \\
& \times\left(N^{-\frac{2}{d}}+N^{-1+\frac{s}{d}}\right)^{\frac{1}{2}}+C \epsilon \\
& \leq C \frac{1+\epsilon^{-\left(\left[\frac{d-s}{2}\right]-\frac{1}{2}\right)\left(1-\frac{d-s}{2}+\left[\frac{d-s}{2}\right]\right)}}{\epsilon^{\left(\left[\frac{d-s}{2}\right]+\frac{1}{2}\right)\left(\frac{d-s}{2}-\left[\frac{d-s}{2}\right]\right)}}\left(N^{-\frac{1}{d}}+N^{-\frac{1}{2}+\frac{s}{2 d}}\right)+C \epsilon .
\end{aligned}
$$

On the other hand, combining (5.9) and (5.6), and then using that $\mu_{X_{N}, \epsilon_{0}}=h \sigma$, (5.1), (5.7) and (5.4), we obtain

$$
\begin{aligned}
\frac{\#\left(D \cap X_{N}\right)}{N}-\frac{\sigma(D)}{\sigma\left(\mathbb{S}^{d}\right)} & \geq \int f_{\epsilon}^{-} d \mu_{X_{N}, \epsilon_{0}}-C \epsilon=\int f_{\epsilon}^{-} h d \sigma-C \epsilon \\
& \geq-C \frac{1+\epsilon^{-\left(\left[\frac{d-s}{2}\right]-\frac{1}{2}\right)\left(1-\frac{d-s}{2}+\left[\frac{d-s}{2}\right]\right)}}{\epsilon^{\left(\left[\frac{d-s}{2}\right]+\frac{1}{2}\right)\left(\frac{d-s}{2}-\left[\frac{d-s}{2}\right]\right)}}\left(N^{-\frac{1}{d}}+N^{-\frac{1}{2}+\frac{s}{2 d}}\right)-C \epsilon
\end{aligned}
$$

In conclusion, we obtain the estimate

$$
\begin{aligned}
\mid \frac{\#\left(D \cap X_{N}\right)}{N} & -\frac{\sigma(D)}{\sigma\left(\mathbb{S}^{d}\right)} \mid \\
& \leq C \frac{1+\epsilon^{-\left(\left[\frac{d-s}{2}\right]-\frac{1}{2}\right)\left(1-\frac{d-s}{2}+\left[\frac{d-s}{2}\right]\right)}}{\epsilon^{\left(\left[\frac{d-s}{2}\right]+\frac{1}{2}\right)\left(\frac{d-s}{2}-\left[\frac{d-s}{2}\right]\right)}}\left(N^{-\frac{1}{d}}+N^{-\frac{1}{2}+\frac{s}{2 d}}\right)+C \epsilon .
\end{aligned}
$$

In order to deal with the right hand side of (5.10), we consider two different cases: $d-2<s<d$ and $0 \leq s \leq d-2$.

Assume first that $\bar{d}-2<s<d$, thus $[(d-s) / 2]=0$. Then, (5.10) leads to

$$
\left|\frac{\#\left(X_{N} \cap D\right)}{N}-\frac{\sigma(D)}{\sigma\left(\mathbb{S}^{d}\right)}\right| \leq C \epsilon^{-\frac{d-s}{4}} N^{-\frac{d-s}{2 d}}+C \epsilon .
$$

Remember that this estimate holds whenever $\epsilon>\epsilon_{0} N^{-1 / d}$, hence we can take

$$
\epsilon=N^{-\frac{2(d-s)}{d(d-s+4)}}
$$

for all $N$ big enough, and then (5.11) yields (5.5).

Let us deal now with the case $0 \leq s \leq d-2$. From (5.10) we get

$$
\left|\frac{\#\left(X_{N} \cap D\right)}{N}-\frac{\sigma(D)}{\sigma\left(\mathbb{S}^{d}\right)}\right| \leq C \epsilon^{-\frac{d-s-1}{2}} N^{-\frac{1}{d}}+C \epsilon
$$

As before, this holds whenever $\epsilon>\epsilon_{0} N^{-1 / d}$, hence we can take $\epsilon=N^{-\frac{2}{d(d-s+1)}}$ for all $N$ big enough, and then (5.12) yields (5.5) . 


\section{REFERENCES}

[1] J. Beck, Sums of distances between points on a sphere - an application of the theory of irregularities of distribution to discrete geometry. Mathematika 31, no. 1, 33-41, 1984.

[2] C. Beltrán, J. Marzo, J. Ortega-Cerdà, Energy and discrepancy of rotationally invariant determinantal point processes in high dimensional spheres, J. of Complexity, 37, 76-109, 2016.

[3] C. Beltrán, N. Corral, J. G. Criado del Rey, Discrete and continuous green energy on compact manifolds, J. Approx. Theory 237, 160-185, 2019.

[4] L. Bétermin. E. Sandier, Renormalized Energy and Asymptotic Expansion of Optimal Logarithmic Energy on the Sphere, Constr. Approx. 47, no. 1, 39-74, 2018.

[5] J.S. Brauchart, Points on an unit sphere in $\mathbb{R}^{d+1}$, Riesz energy, discrepancy and numerical integration. PhD thesis, Institut für Mathematik A, Technische Universität Graz, Graz, Austria, 2005.

[6] J.S. Brauchart. Optimal logarithmic energy points on the unit sphere. Math. Comp. 77, no. 263, 1599-1613, 2008.

[7] J. S. Brauchart, D. P. Hardin, E. B. Saff. The next-order term for optimal Riesz and logarithmic energy asymptotics on the sphere. Recent advances in orthogonal polynomials, special functions, and their applications, 31-61, Contemp. Math., 578, Amer. Math. Soc., Providence, RI, 2012.

[8] J. S. Brauchart, P.J. Grabner. Distributing many points on spheres: minimal energy and designs. J. Complexity 31, no. 3, 293-326, 2015.

[9] I. Chavel, Riemannian Geometry. A Modern Introduction. Second Edition. Cambridge Studies in Advanced Mathematics, 2006.

[10] B. E. J. Dahlberg, On the distribution of Fekete points, Duke Math. J. 45, no. 3, 537-542, 1978.

[11] F. Dai, Y. Xu, Approximation theory and harmonic analysis on spheres and balls, Springer Monographs in Mathematics. Springer, New York, 2013.

[12] S. B. Damelin, V. Maymeskul, On point energies, separation radius and mesh norm for s-extremal configurations on compact sets in $\mathbb{R}^{n}$, J. of Complexity 21, 6, 845-863, 2005.

[13] P. D. Dragnev, E. B. Saff, Riesz Spherical Potentials with External Fields and Minimal Energy Points Separation. Potential Anal. 26, 139-162, 2007.

[14] A. Erdélyi, W. Magnus, F. Oberhettinger, F. G. Tricomi. Tables of integral transforms. Vol. II. McGraw-Hill Book Company, Inc., New York-Toronto-London, 1954.

[15] M. Götz, On the distribution of weighted extremal points on a surface in $\mathbb{R}^{d}, d \geq 3$. Potential Anal. 13, no. 4, 345-359, 2000.

[16] W. Kleiner, Degree of convergence of the extremal points method for Dirichlet's problem in the space. Colloq. Math. 12, 41-52, 1964.

[17] J. Korevaar, Fekete extreme points and related problems, Approximation Theory and Function Series, Bolyai Soc. Mathematical Studies, 5, 35-62, 1996.

[18] A. Kuijlaars, E. B. Saff. Asymptotics for minimal discrete energy on the sphere. Trans. Amer. Math. Soc.. 350, no. 2, pp. 523-538, 1998.

[19] A. Kuijlaars, E. B. Saff, X. Sun, On separation of minimal Riesz energy points on spheres in Euclidean spaces, J. Comput. Appl. Math. 199, 1, 172-180, 2007.

[20] S. Lang, Introduction to Arakelov Theory, Springer-Verlag New York, 1988.

[21] A. Lubotzky, R. Phillips, P. Sarnak, Hecke operators and distributing points on the sphere. I. Frontiers of the mathematical sciences: 1985 (New York, 1985). Comm. Pure Appl. Math. 39 , no. S, suppl., S149-S186, 1986.

[22] R. Peyre, Non-asymptotic equivalence between $W^{2}$ distance and $\dot{H}^{-1}$, to appear in ESAIM, COCV, 2018.

[23] E.A. Rakhmanov, E. B. Saff, Y.M. Zhou. Minimal discrete energy on the sphere. Math. Res. Lett. 1, no. 6, 647-662, 1994. 
[24] S. Serfaty, Coulomb Gases and Ginzburg-Landau Vortices, Zurich Lectures in Advanced Mathematics, EMS, 2015.

[25] P. Sjögren, Estimates of mass distributions from their potentials and energies, Ark. Mat. 10, 59-77, 1972.

[26] P. Sjögren, On the regularity of the distribution of the Fekete points of a compact surface in $\mathbb{R}^{n}$. Ark. Mat. 11, 147-151, 1973.

[27] C. Villani, Topics in Optimal Transportation, Graduate Studies in Mathematics, American Mathematical Society, 2003.

[28] G. Wagner, On means of distances on the surface of a sphere. II. Upper bounds, Pacific J. Math. 154, no. 2, 381-396, 1992.

[29] T. Wolff, Fekete points on spheres, manuscript.

J. MARZO

Departament de Matemàtiques i Informàtica, Barcelona Graduate School of Mathematics (BGSMath), Universitat de Barcelona, Gran Via 585, 08007 Barcelona, Spain

E-mail address: jmarzo@ub.edu

A. MAS

Departament de Matemàtiques, Universitat Politècnica de Catalunya, Campus Diagonal Besòs, Edifici A (EEBE), Av. Eduard Maristany 16, 08019 Barcelona, Spain

E-mail address: albert.mas.blesa@upc.edu 